\title{
Instruktion und Verständigung unter fragilen Interaktions- bedingungen: Gesprächsanalytische Untersuchungen zu beruflichen Qualifizierungsmaßnahmen für Flüchtlinge
}

\begin{abstract}
Der vorliegende Aufsatz untersucht Ausbildungsinteraktionen in zwei beruflichen Qualifizierungsmaßnahmen für Flüchtlinge. Solche Maßnahmen werden seit 2015 verstärkt angeboten, um die Geflüchteten möglichst umfassend und zügig auf eine Arbeitsaufnahme in Deutschland vorzubereiten. Im Kontext einer ethnografischen Studie untersuchen wir mit Methoden der multimodalen Interaktionsanalyse, a) wie in Anleitungsgesprächen Verständigungsprobleme zwischen deutschen Anleitern und auszubildenden Flüchtlingen entstehen und b) welche sprachlich-kommunikativen Praktiken zu ihrer Bearbeitung eingesetzt werden. Dabei lassen sich ebenso gelungene wie Probleme erzeugende Kommunikationspraktiken feststellen. Da die meisten Geflüchteten zu Beginn der untersuchten Maßnahmen noch keine Integrationskurse besucht hatten und nur über wenige Deutschkenntnisse verfügten, liegt der primäre Fokus der Analyse auf der Beteiligungsweise der Ausbilder, betrachtet diese aber im sequenziellen Kontext der Interaktionsbeteiligung der auszubildenden Flüchtlinge. Die Untersuchung beruht auf 22 Stunden Videoaufnahmen praktischer Ausbildungen.

This article reports on a study of instructional interaction in two professional training measures for refugees. This kind of measure is now widely used in Germany in order to prepare refugees for the German labour market. Based on ethnographic fieldwork, we use a multimodal interaction approach to analyse video-recorded instructional sequences. Our study focuses a) on how problems of mutual understanding develop in these interactions between the German instructors and the refugees and $b$ ) on the practices the participants use to deal with these problems. On the basis of our findings we discuss which practices foster mutual understanding and which practices are likely to become sources of problems for the accomplishment of intersubjectivity. Most of the refugees included in the study had not yet taken part in an "integration course" (which is deemed to support the learning of German as a second language up to level CEFR-B1). Therefore, our study focuses mainly on the instructors' actions, analysing them in the sequential context of the interaction with the refugees. The study draws on 22 hours of video-recordings of practical instructions in workplace settings (wood-and metal-working, cooking).
\end{abstract}

\section{Einleitung ${ }^{1}$}

Im vorliegenden Aufsatz untersuchen wir Ausbildungsinteraktionen in zwei beruflichen Qualifizierungsmaßnahmen, die wir im Rahmen des IDS-Projekts „Deutsch im Beruf: Die sprachlich-kommunikative Integration von Flüchtlingen" wissenschaftlich begleitet haben. Wie in der Einleitung zu diesem Themenheft ausgeführt, startete unser Projekt zu Beginn des Jahres 2016, also kurz nachdem die große Gruppe der Fluchtmigranten 2015 nach Deutschland kam. ${ }^{2}$ Wir konnten den Integrationsprozess der Flüchtlinge von Anfang an dokumentieren und die wichtigsten Integrationsetappen bezüglich ihrer sprachlichen und kommunikativen Anforderungen und Abläufe erfassen und analysieren. Neben den sprachlichen Integrationskursen (Hünlich/Wolfer/Lang in diesem Heft) sind es vor allem die vielfältigen, bundesweit angebotenen beruflichen Qualifizierungsmaßnahmen, in

Wir danken zwei anonymen Gutachtern für Hinweise und Kommentare zur ersten Fassung des Textes.

2 In diesem Beitrag wird ausschließlich die männliche Personenbezeichnung verwendet, es können damit aber auch beide Geschlechter gemeint sein. 
denen die ersten wichtigen Weichen für die berufliche und soziale Integration der Flüchtlinge gestellt werden. ${ }^{3}$ Deshalb haben wir uns dafür entschieden, zwei solcher Maßnahmen längsschnittlich wissenschaftlich zu begleiten. Unsere Untersuchung richtet sich also auf die mündliche Kommunikation in beruflichen Ausbildungskontexten. Im Zentrum unseres Interesses stehen die sprachlichen und kommunikativen Anforderungen in Ausbildungsinteraktionen und die Praktiken, die die Beteiligten, Ausbilder wie auszubildende Flüchtlinge zur Bearbeitung dieser Anforderungen einsetzen. Besonderes Augenmerk liegt naturgemäß auf den Problemen, die durch mangelnde Sprachbeherrschung, Verständigungsschwierigkeiten und erwartungsinkongruente Interaktionspraktiken entstehen.

Die berufliche Qualifizierung und Vermittlung anerkannter Asylbewerber ist Aufgabe der Jobcenter. Für Asylbewerber bzw. Geduldete ist die Bundesagentur für Arbeit (BA) zuständig. Die von uns begleiteten Maßnahmen „Perspektive für Flüchtlinge“ („PerF Plus“) und „GASTRO ${ }^{4}$ sind zwei von vielen Qualifizierungsprojekten, die seit 2015 für Asylbewerber konzipiert wurden. Ziel solcher Maßnahmen ist es, die Geflüchteten möglichst umfassend und zügig auf eine Arbeitsaufnahme in Deutschland vorzubereiten, indem fachliche Kenntnisse, Fähigkeiten und Fertigkeiten festgestellt und Sprachkenntnisse vermittelt werden. ${ }^{5}$ Den rechtlichen Rahmen solcher Maßnahmen bildet $\$ 45$ des Sozialgesetzbuches (Drittes Buch). Da die Bundesagentur rechtlich gesehen keine allgemeine Deutschförderung finanzieren darf, beschränkt sich die Vermittlung von Deutschkenntnissen in allen Maßnahmen auf berufsbezogenes Deutsch.

Die beiden von uns untersuchten Maßnahmen „PerF Plus“ und „GASTRO“ unterscheiden sich in vielerlei Hinsicht. Wir haben für unsere Untersuchung bewusst nach zwei maximal kontrastierenden Maßnahmen gesucht, da wir den möglichen Einfluss von unterschiedlichen äußeren Faktoren auf die Binnenstruktur der Kommunikation erfassen und analysieren wollten. Die „PerF Plus"-Maßnahme wurde in einer bayrischen Kleinstadt im ländlichen Raum angeboten, wo es nur wenige Anbieter von Integrationskursen gibt und die Flüchtlinge deshalb sehr lange auf einen Sprachkurs warten müssen. Hier war davon auszugehen, dass viele Geflüchtete ohne vorherige Deutschkurse berufliche Qualifizierungsmaßnahmen besuchen. „PerF Plus" gehört zu den Projekten, die von der Bundesagentur in Bayern flächendeckend angeboten werden. Struktur und Ablauf der Maßnahme sind von der Agentur vorkonzipiert. Die praktischen Ausbilder sind für Aspekte des Spracherwerbs und der Interkulturalität der Kommunikation meist nicht (vor-)geschult bzw. erfahren. „PerF Plus“ ist nicht spezifisch auf die beruflichen Kenntnisse und Bedürfnisse der Teilnehmer zugeschnitten, sondern wird entsprechend der wirtschaftlichen, industriellen und arbeitsmarktspezifischen Gegebenheiten der jeweiligen Region gestaltet.

In ihrer Statistik zum Jahr 2017 hält die Bundesagentur für Arbeit fest, dass im betreffenden Jahr insgesamt 673.000 Personen aus den acht nichteuropäischen Asylherkunftsländern ihre Arbeitslosigkeit beenden konnten, aber nur 70.000 (also 10\%) auf dem 1. Arbeitsmarkt (inkl. Selbständigkeit) beschäftigt waren (Bundesagentur für Arbeit 2017, S. 10). Demgegenüber befanden sich 421.000 Personen (63\%) aus dieser Gruppe in diversen Fördermaßnahmen.

4 Im Gegensatz zu den „PerF Plus“-Maßnahmen, die in ganz Bayern angeboten werden, handelt es sich bei „GASTRO“ um eine singuläre berufliche Qualifizierungsmaßnahme, weshalb wir sie anonymisiert haben.

5 Auch in den beiden von uns untersuchten Maßnahmen gab es neben dem praktischen Unterricht separaten Deutschunterricht. Da wir aber nur in einer der Maßnahmen den Deutschunterricht filmen durften, können wir leider auf die Durchführung der Sprachkurse und die Verzahnung der Praxismaßnahme mit der Sprachförderung nicht eingehen. 
Dagegen wurde die Maßnahme „GASTRO“ im städtischen Raum der Metropolregion Rhein-Neckar durchgeführt. In urbanen Gebieten werden in der Regel mehr Integrationsbzw. Sprachkurse angeboten, sodass man bei „GASTRO“ im Gegensatz zu „PerF Plus“ davon ausgehen konnte, dass ein Teil der Teilnehmer einen Deutschkurs besucht hat. „GASTRO“ wurde von zwei engagierten Menschen aus dem Bereich der Hotellerie/Gastronomie selbst initiiert, geplant und dann bei der Arbeitsagentur beantragt. Die Maßnahme wurde von Anfang an nur für Flüchtlinge angeboten, die auch im gastronomischen Bereich arbeiten wollten. Die Projektleiter (FN und UH) haben große praktische Erfahrungen im Bereich der interkulturellen Kommunikation und des Spracherwerbs im Erwachsenenalter: FN ist seit über 30 Jahren Gastronom, der schon an vielen Orten auf der Welt gearbeitet und früher ein ähnliches Qualifizierungsprojekt in Lateinamerika realisiert hat. Unterstützt wird er von UH, Sprachlehrerin an einer Hotelfachschule, die sich im ehrenamtlichen Bereich für Flüchtlinge engagiert. Die beiden Maßnahmen unterscheiden sich also in den äußeren Rahmenbedingungen stark voneinander. In den empirischen Interaktionsanalysen werden wir untersuchen, ob und wie diese Kontextfaktoren auch in den face-to-face Kommunikationssituationen der praktischen Ausbildung eine Rolle spielen.

\section{Migration, Sprache, Arbeit und institutionelle Settings}

Die Arbeitswelt und daher auch die Kommunikation am Arbeitsplatz sind nicht erst seit der Fluchtmigration, sondern seit geraumer Zeit durch Migration und Globalisierung geprägt. ${ }^{6}$ Spätestens seit den 2000 er Jahren sind in vielen Ländern und Regionen der Welt „superdiverse“ Gesellschaften (Vertovec 2007) entstanden, die durch folgende Merkmale gekennzeichnet sind: a) zunehmende Vielfalt von Herkunftsländern (in Städten wie London oder Frankfurt leben Menschen aus über 170 Ländern (ebd., S. 1029; Römhild/Vertovec 2009, S. 41)); b) zunehmende Vielfalt im Rechtsstatus und der sozialen Lagen (eingebürgerte Migranten, Migranten mit sicheren oder befristeten Aufenthaltstiteln, „illegale“ Migranten etc.) und c) zunehmende Vielfalt von Migrationspfaden (unterschiedliche Formen der Arbeitsmigration, Flucht und Asyl, Familienzusammenführung, Bildungsmigration, Pendel- und Transmigration etc.) (ebd., S. 38). Die Entwicklung superdiverser Gesellschaften hat auch in sprachlicher Hinsicht vielerlei Spuren hinterlassen, die von der germanistischen Soziolinguistik gut erforscht sind wie etwa die Herausbildung von polyethnolektalen Sprechstilen (Wiese 2012; Auer 2013), neuere Formen der Transmigration und sprachlicher Variation (Cindark/Keim 2016) oder die Sichtbarkeit sprachlicher Diversität im öffentlichen Raum (Cindark/Ziegler 2016). Während die informelle Mündlichkeit in diversen Migrantenmilieus (Keim 2008; Cindark 2010) gut erforscht ist, hat die Untersuchung der Kommunikation in beruflichen Kontexten in Deutschland bisher kaum eine Rolle gespielt.

$\mathrm{Zu}$ den wenigen Untersuchungen zählt das Projekt „Deutsch am Arbeitsplatz“, das von 2007 bis 2013 vom Deutschen Institut für Erwachsenenbildung durchgeführt wurde. Ein Ergebnis der Projektarbeit sind zehn ethnografische Firmenportraits, die die Rolle der Sprache und Kommunikation im betrieblichen Alltag nachzeichnen. Hier konnten etwa festgestellt werden: die gestiegene Bedeutung kommunikativer Kompetenzen als konstitutiver Bestandteil der beruflichen Handlungsfähigkeit, wachsende Anforderungen an

Wie Hazel/Svennevig (2018, S. 1f.) zu Recht ausführen, gibt es die arbeitsbasierte Kommunikation von Menschen mit unterschiedlichen Erstsprachen schon seit der Antike. 
Schriftsprachlichkeit, erhöhte Ansprüche an das („lebenslange“) Lernen am Arbeitsplatz als Folge des Strukturwandels und häufig mehrsprachige Belegschaften, deren Multilingualität aber in Interviews mit Führungskräften im betrieblichen Alltag nicht immer positiv wertgeschätzt wurde (Grünhage-Monetti/Svet 2014, S. 182-190). Neben den Firmenporträts führte das Projektteam „sprachdidaktische Analysen der in Schlüsselszenarien beruflicher Praxis erfassten Texte (mündliche als auch schriftliche)“ (ebd., S. 190) durch. In den Publikationen der Projektgruppe (Grünhage-Monetti 2009; Berg/Grünhage-Monetti 2009; Grünhage-Monetti 2013) ist allerdings nicht zu erkennen, wie diese Sprachanalysen vorgenommen wurden. Als Ergebnisse hält das Projektteam neben der Relevanz des Fachwortschatzes und der gestiegenen Bedeutung der Schriftsprachlichkeit insbesondere fest: Im Arbeitsalltag werden Migranten mit kompetenzniveau-übergreifenden sprachlichen Anforderungen (i.S. des GER) konfrontiert, worauf sie „durch Sprachkurse und Unterrichtskommunikation nicht vorbereitet sind“ (Grünhage-Monetti/Svet 2014, S. 192). Andererseits sind die Autorinnen aber auch der Meinung, dass durch Sprachsensibilisierungen die muttersprachlichen Interaktionspartner geschult werden können, um in Kontakt mit Migranten arbeitsplatzrelevante Sprachhandlungen auf Anfänger-Niveaustufen (A1, A2) zu produzieren (ebd.).

In der deutschsprachigen gesprächsanalytischen Forschung kommen Migranten am Arbeitsplatz bisher fast nur als Klienten von Institutionen, z.B. in der Behördenkommunikation (Rosenberg 2014) oder in Polizeivernehmungen (Hee 2012), aber kaum einmal selbst als berufliche Akteure vor. Ausnahmen sind lediglich Untersuchungen zum Gesundheitswesen, etwa von Schön (2012) zu ausländischen Ärzten und deutschen Patienten und von Meyer (2004) zum Adhoc-Dolmetschen durch migrantische Krankenschwestern, aus denen auch entsprechende Trainingsprogramme entwickelt wurden (Meyer et al. 2010). In der internationalen Forschung wurden dagegen bereits seit den 1970er Jahren Studien zum Themenfeld „Migration, Sprache, Arbeit und institutionelle Settings" durchgeführt, auch wenn diese oft „for educational intervention rather than for research" (Roberts 2007, S. 405) konzipiert wurden. Die wichtigsten Forschungsfragen waren: Wie funktioniert die Verwendung der Landessprache als Interaktionssprache zwischen Minderheits- und Mehrheitsangehörigen (Gumperz 1982; Gumperz/Roberts 1991) und ihr ungesteuerter Erwerb als L2 (Svennevig 2015, 2018) im Arbeitskontext? In welchem Maße führen die Verwendung der Mehrheitssprache und weitere sprachliche und kommunikative Praktiken im Kontext Arbeit/Ausbildung zur Reproduktion sozialer Ungleichheit und Diskriminierung (Erickson/Shultz 1982; Roberts/Davies/Jupp 1992; Sarangi/Roberts 1999; Roberts 2013; Tranekjær 2015)? Welche Rollen spielen Lingua Franca und multilinguale Kommunikationspraktiken (Clyne 1994; Mondada 2004; Lüdi/Höchle/Yanaprasart 2010) in interethnischen und -kulturellen Arbeitssituationen?

Gumperz (1982) hat als Pionier der interkulturellen Kommunikationsforschung aus interaktionaler Perspektive bereits früh herausgearbeitet, dass die grammatisch und lexikalisch richtige Verwendung der Landessprache durch Minderheitsangehörige in face-toface Interaktionen mit Mehrheitsangehörigen noch keine Garantie für intersubjektives Verstehen darstellt. Aufgrund unterschiedlicher kommunikativer Konventionen in den jeweiligen Codes (z.B. Britischenglisch vs. Indischenglisch im Rahmen eines Bewerbungsgesprächs, siehe Gumperz/Jupp/Roberts 1979, S. 5) können etwa die Gliederung von Informationen, Strukturierung von Argumentationen, spezifische Mittel der Kooperation, Formen der Selbstdarstellung in der Interaktion etc. sehr unterschiedlich signali- 
siert und verstanden werden. ${ }^{7}$ Diese Befunde waren der Ausgangspunkt seiner Theorie der Kontextualisierung (Gumperz 1982), nach der die jeweiligen Codes über spezifische konventionalisierte cues verfügen, mit denen Aspekte wie Relevanz einer Mitteilung, Sprechakttyp, Einstellungen zum Gegenüber, Bewertungen, Identitätsansprüche oder Emotionen kontextualisiert werden. Wenn diese Konventionen nicht geteilt oder gekannt werden, können sie in der Kommunikation zwischen Mehrheits- und Minderheitsangehörigen zu Missverständnissen führen (Gumperz 1982, S. 173; Hinnenkamp 1989, S. 11 für Missverständnisbeispiele aufgrund erwartungsinkongruenter Intonationsmuster in Dienstleistungsinteraktionen).

Nicht- und Missverstehen aufgrund unterschiedlicher kulturspezifischer Kontextualisierungskonventionen haben gerade bei der Arbeitskommunikation weitreichende Folgen. Insbesondere in Gatekeeping-Situationen wie Berufsberatungen, Bewerbungsgesprächen und Praktika, die für den professionellen Werdegang des Einzelnen von entscheidender Bedeutung sind, können sie zu Ungleichbehandlung und Diskriminierung führen. So konnten Erickson/Shultz (1982, S. 132) in ihrer Studie zu Beratungssitzungen an amerikanischen Hochschulen feststellen, dass white counselor einige schwarze Bewerber u.a. deswegen nicht für ein Hochschulstudium empfohlen haben, weil diese im Beratungsgesprächen deutlich weniger Rückmeldesignale gaben (wie etwa Blickkontakt suchen, nicken, Diskurspartikel wie „yeah“ einstreuen) und somit aus der Erwartungshaltung der weißen Berater betrachtet keine aktive Zuhörerschaft und Desinteresse signalisierten. Die divergierenden diskursiven Praktiken und unausgesprochenen kommunikativen Erwartungen hebt auch Roberts (2013) als größte Hindernisse für Minderheitsangehörige in Bewerbungsgesprächen hervor. In ihrer Analyse von Vorstellungsgesprächen im Niedriglohnsektor zeigt sie auf, dass von den Bewerbern erwartet wird, nicht nur Interesse für die monotone Arbeit zu zeigen, sondern dass sie auch selbstorganisiert, -bewusst und reflektiert genug sind, um zu formulieren, wie sie den Anforderungen der repetitiven Arbeit gerecht werden wollen (ebd., S. 86). Da die migrantischen Anwärter auf diese versteckten konventionalisierten Erwartungen häufig nicht die präferierten Antworten liefern, fallen sie hier durch (ebd., S. 88): „Candidates may well be competent for the job but they are excluded from it because they are not competent for the interview" (ebd., S. 92). Direkte Formen der Ausgrenzung analysiert Tranekjær (2015) in Auswahlgesprächen für Praktika in Dänemark. Die Autorin zeigt auf, wie nationale, sprachliche und religiöse Stereotypisierungen seitens der Interviewer zum „Othering“ der Bewerber führen. Wenn die Interviewer auf nationale/kulturelle Unterschiede zu sprechen kommen, wird die „dänische Art" oft positiv charakterisiert, der nationale und kulturelle Hintergrund der Minderheitsangehörigen dagegen tendenziell negativ (ebd., S. 127-162).

Schließlich untersuchen eine Reihe von Arbeiten multilinguale Praktiken und die Verwendung von Englisch als Lingua Franca, durch die in Zeiten der Globalisierung Arbeitsplätze zunehmend gekennzeichnet sind (Clyne 1994; Mondada 2004; Lüdi/Höchle/Yanaprasart 2010; House 2010). Lüdi/Höchle/Yanaprasart (2010) untersuchen das Sprachenmanagement eines international agierenden Pharmaunternehmens in Basel. Ein Manager des Unternehmens sagt im Interview, das Projektteam „,would not make interesting findings because «our corporate language is English»" (ebd., S. 220). Die Untersuchung der tatsächlichen Sprachverwendung im Betrieb offenbart jedoch eine plurilinguale Kommuni-

Zu kulturell unterschiedlichen Konventionen in intraethnischen Kommunikationssituationen siehe auch die Untersuchung zu Ost- und Westdeutschen in Bewerbungsgesprächen (Kern 2000). 
kationspraxis, in der sowohl Englisch als auch Französisch und Deutsch vorkommen (ebd., S. 223).

\section{Verstehen im Gespräch}

Der Fokus unserer Untersuchung liegt auf Instruktionsinteraktionen. Wir untersuchen Erstinstruktionen, in denen Praktikanten bestimmte Handlungsvollzüge erstmals erklärt und demonstriert werden. Die Instruktion hat insofern immer einen deskriptiven Aspekt (wie etwas gemacht werden soll), während der unmittelbar auffordernde (dass die instruierte Tätigkeit im unmittelbaren Anschluss ausgeführt werden soll) nicht immer hinzu tritt (vgl. Lindwall/Lymer/Greiffenhagen 2015). Dies spiegelt sich in unterschiedlichen sprachlichen Instruktionsformaten, z.B. der Verwendung von Deklarativen für Erstinstruktionen vs. Imperativen für wiederholte und korrektive Instruktionen wider (Deppermann 2018). Instruktionen praktischer Handlungen sind nicht nur verbale Handlungen, sondern beinhalten auch leibliche Demonstrationen der zu lernenden Fertigkeit (Lindwall/ Ekström 2012) oder gestische Demonstrationen (Deppermann 2016).

Ein wesentlicher Aspekt des Instruierens, zumal im Kontext der L1-L2-Interaktion, ist die Verständigungssicherung. Die bloße Behauptung von Verstehen (Sacks 1992) durch die Instruktionsempfänger, etwa in Form von Rückmeldesignalen oder Wiederholungen, ist gerade im Kontext von Lehr-Lern-Interaktionen ein sehr problematischer Indikator für Verstehen, da durch solche Reaktionen nicht klar wird, ob wirklich verstanden, geschweige denn, ob etwas gelernt wurde (Koole 2012). Gelungene Verständigungssicherung muss sich daher an belastbareren Indikatoren bemessen, etwa der Äußerung von Schlussfolgerungen, weiterführenden Fragen oder der leiblichen Demonstration des Verstandenen (vgl. Deppermann/Schmitt 2008, Hindmarsh/Raynolds/Dunne 2011). Diese Überlegungen zeigen bereits, dass Verstehen für den konversationsanalytischen Ansatz kein Prozess ist, der nur durch Einsicht in die mentalen Vorgänge des Rezipienten zu prüfen wäre, sondern dass es als sozialer, beobachtbar angezeigter Vorgang begriffen und untersucht wird - und in genau dieser Weise ist es auch für die Interaktionsteilnehmer relevant und folgenreich. Grundsätzlich hat dabei die Herstellung von Verständigung (Intersubjektivität) eine zumindest dreischrittige Sequenzstruktur: a) Akteur A produziert eine Handlung, die von B verstanden werden soll; b) B zeigt sein Verstehen (mehr oder weniger explizit) durch eine Reaktion an; c) A zeigt in seiner Reaktion auf B an, ob er Bs Reaktion als angemessene Antwort auf sein Handeln und damit als hinreichendes Verständnis seines Handelns im ersten Schritt akzeptiert (vgl. Deppermann 2008). Verstehen wird allerdings oft schon während der Produktion eines Turns vom Rezipienten durch nonverbale Reaktionen angezeigt (Mondada 2011). Verstehen bedarf also der beobachtbaren Kundgabe und der interaktiven Ratifikation, um als interaktiv gültig behandelt zu werden. In Prozessen gelungener Verständigung baut sich ein common ground zwischen den Gesprächsteilnehmern auf, den diese für ihre folgenden Handlungen voraussetzen können (Clark 1992). Wichtig dafür ist der Adressatenzuschnitt von Äußerungen (recipient design), mit dem die speziellen Vorerfahrungen mit dem Adressaten, insbesondere seine Wissensvoraussetzungen und Erwartungen in der Interaktionssituation bei der Formulierung in Rechnung gestellt werden. Dies erfordert sowohl die genaue Beobachtung des Interaktionshandelns des Partners als auch weitergehende Hypothesen über fachliches Wissen, Sprachkenntnisse und andere Orientierungen der Interaktionspartner (Deppermann/Schmidt 2016). Während Nichtverstehen vom Rezipienten unmittelbar angezeigt werden kann und (damit) Reparaturen ini- 
tiiert werden (Schegloff/Jefferson/Sacks 1977), zeigt sich Missverstehen oft erst viel später. Es kann entweder vom Produzenten des Verstehensgegenstands anhand unpassender Reaktionen des Rezipienten identifiziert werden oder aber auch von Letzterem selbst aufgrund folgender inkongruenter Informationen und Handlungen seines Gegenübers erschlossen werden (Hinnenkamp 1998).

Für die Interaktion zwischen L1- und L2-Sprechern gelten oft erschwerte Verstehensbedingungen. Bisherige Forschungen haben gezeigt, dass Interaktionsteilnehmer in diesen Situationen bestimmte Strategien der Verständigungssicherung verstärkt einsetzen, die auch sonst zu beobachten sind, wenn Verständigung prekär ist oder bereits gescheitert war. Dazu gehören der Einsatz von Verstehensprüfungen und -nachfragen, Fremdreparaturen und Hilfen bei Formulierungsproblemen (Dausendschön-Gay 1988; Kurhila 2001, 2006; Kameyama 2004; Svennevig 2018), Worterklärungen und Definitionen (Mazeland/Zaman-Zadeh 2004; Mortensen 2011; Fasel Lauzon 2014), Selbstreformulierungen, vereinfachte Lexik und Syntax, Linksversetzungen (Svennevig et al. 2017), Fremdreformulierungen und metadiskursive Kommentare (Dausendschön-Gay/Gülich/Krafft 1995), Rückversicherungspartikeln und die Einforderung von Rückmeldungen, Wiederholungen (Svennevig 2004, 2018), die Unterteilung von Turns in kleine Einheiten (installments), die didaktische, deutliche (Vor-)Strukturierung von langen Turns (Svennevig 2015), überartikulierte und langsame Sprechweise sowie illustrative Gesten (Mori/Hayashi 2006). Verstehen und Verständigungssicherung stehen allerdings zumindest potenziell in einem Konkurrenzverhältnis zu interpersoneller Anerkennung (face-work, vgl. Goffman 1955): Sowohl die Anzeige nicht verstanden zu haben als auch die Korrektur von Missverständnissen werden häufig vermieden, um nicht das eigene Gesicht bzw. das des Gesprächspartners zu beschädigen (Kurhila 2006), was wiederum die Sicherstellung von Intersubjektivität und die Bereinigung von Verständigungsproblemen beeinträchtigen kann.

\section{Interaktionsanalysen zu zwei beruflichen Qualifizierungsmaßnahmen für Flüchtlinge}

Bei den beruflichen Maßnahmen handelt es sich um Kommunikationssituationen, die der Gesprächsgattung Lehr-Lerninteraktionen bzw. Instruktionsgespräche zuzuordnen sind. Bis auf wenige Teilnehmer verfügte der Großteil der Geflüchteten nur über geringe Deutschkompetenzen, da sie vor dem Beginn der Maßnahmen noch keinen Integrationskurs besucht hatten. Daher können sich die Ausbilder nicht nur auf ihre primäre Aufgabe, den Praktikanten berufliche Kenntnisse zu vermitteln, konzentrieren, sondern sie werden immer wieder mit Verständigungsproblemen konfrontiert. Sie müss(t)en also bei ihren (Sprach-)Handlungen stets auch die fehlenden Deutschkenntnisse der Auszubildenden in Betracht ziehen.

Im Zentrum unserer Interaktionsanalysen steht daher die Frage, welche sprachlichen und kommunikativen Praktiken in Anleitungsgesprächen zur Verständigungssicherung eingesetzt werden und welche zu Verständigungsproblemen führen. Unser Fokus liegt damit primär auf den Handlungen der Ausbilder, betrachtet diese aber im sequenziellen Kontext der Interaktionsbeteiligung der auszubildenden Flüchtlinge. Dabei sind folgende Aspekte von besonderer Relevanz:

- Wie werden die Instruktionen von den Ausbildern sprachlich und non-verbal gestaltet? Welche Instruktionsformate unterstützen das Verstehen und welche sind eher kontraproduktiv? Welche Rollen spielen dabei linguistische Strukturen wie syntakti- 
sche Komplexität und Fachsprache, aber auch paralinguistische Merkmale wie Sprechtempo, Intonation und Gliederungssignale (Pausen)?

- Wie macht sich Nicht-Verstehen bzw. Missverstehen auf Seiten der Flüchtlinge in der Interaktion bemerkbar?

- Zeigen Ausbilder ein auf die spezifischen (In-)Kompetenzen der Flüchtlinge abgestimmtes recipient design? Mit welchen Praktiken reagieren sie auf Verstehensprobleme?

- Welche Rolle spielt die Gestik in der Verständigung? Wie werden deiktische und ikonische Gesten eingesetzt, um die sprachliche Instruktion zu stützen?

- Welche Rolle spielt Mehrsprachigkeit in den Ausbildungsinteraktionen? Nutzen die Ausbilder mehrsprachige Ressourcen zur Verständnissicherung in der Interaktion?

- Berücksichtigen die Ausbilder, dass einzelne Flüchtlinge im Kurs über mehr Deutschkompetenzen verfügen als andere und daher als Dolmetscher eingesetzt werden könnten?

- Wie gehen die Ausbilder mit der Herausforderung um, neben der Fachvermittlung auch Sprachvermittlung zu betreiben? Benutzen sie Strategien, die speziell auf diese Aufgabe zugeschnitten sind?

Datengrundlage der folgenden Interaktionsanalysen sind 22 Stunden Videoaufnahmen, die im Rahmen unserer Ethnografie der praktischen Ausbildungskontexte erhoben wurden. ${ }^{8}$

\subsection{Maßnahme „PerF Plus“}

„Perspektive für Flüchtlinge Plus“ („PerF Plus“) ist eine von der Bundesagentur für Arbeit in Bayern konzipierte berufliche Maßnahme. Die Maßnahme erstreckt sich über sechs Monate. Entsprechend der Vorgaben der Bundesagentur sollen die Träger in der ersten Phase eine Woche für eine „Standortbestimmung“ und „Bedarfsanalyse" und weitere knapp zwei Wochen für eine „Eignungsfeststellung“ der Teilnehmer vorsehen. Dabei sollen der Bildungsstand, der bisherige berufliche Werdegang und die vorhandenen Kenntnisse und Fähigkeiten erfasst und eine Förder- und Integrationsstrategie abgestimmt werden. In der zweiten Phase steht dann die Kenntnisvermittlung in praktischen Unterrichtseinheiten, die zumeist als Werkstattunterricht stattfinden, im Vordergrund. Im Anschluss soll die Anwendung der erlernten Fähigkeiten in sechswöchigen betrieblichen Praktika vertieft werden. Schließlich sind am Ende der Maßnahme zwei weitere Wochen für Bewerbungen und die Vermittlung in Arbeit vorgesehen.

Die von uns untersuchte „PerF Plus“-Maßnahme ging von Juni bis Dezember 2016. ${ }^{9}$ Aufgrund der wirtschaftlichen und arbeitsmarktspezifischen Gegebenheiten im Umkreis der Kleinstadt waren für die Kenntnisvermittlung in den Werkstätten des Kurses die Fachbe-

8 In der ethnografischen Begleitung der beiden Maßnahmen „PerF Plus“ und „GASTRO“ führten wir sowohl Einzel- als auch Gruppeninterviews durch. Interviewpartner waren neben den Teilnehmern auch die Kursleiter, die Ausbilder in den Fachbereichen, die Anbieter der Maßnahmen sowie die Vertreter der Bundesagentur auf lokaler und regionaler Ebene. Wir besuchten Teilnehmer in ihren Unterkünften und sprachen mit ihren ehrenamtlichen Helfern, um die sozialen Faktoren einordnen zu können, die die Maßnahmen beeinflussen. Ausführliche ethnografische Darstellungen finden sich in Cindark/Hünlich (2017) und Cindark (im Dr.).

9 Unsere Videoaufnahmen von den Praktika betragen insgesamt knapp zehn Stunden. Der Maßnahmenträger gab uns die Zustimmung für Aufnahmen in der Metall- und Holzwerkstatt. Die Zeitpunkte, zu denen wir Videoaufnahmen machen konnten, waren etwa in der Mitte der Praktikumszeit angesiedelt und wur- 
reiche Metall, Bau sowie Soziales/Gesundheit vorgesehen. In der Maßnahme waren anfangs 20 Teilnehmer. Durch den Ausstieg von einigen und das Nachrücken neuer Teilnehmer besuchten insgesamt 23, ausschließlich männliche Flüchtlinge den Kurs. Der Großteil der Teilnehmer stammte aus Syrien, drei kamen aus dem Irak.

Hinsichtlich der erforderlichen Deutschkompetenzen der Teilnehmer hält die Bundesagentur für Arbeit fest: „Die Teilnehmer sollen über Sprachkenntnisse verfügen, die es zulassen, den Inhalten der Maßnahme zu folgen. Dies ist in der Regel nach der Teilnahme an einem Integrationskurs der Fall." ${ }^{10}$ Die Bundesagentur und somit auch die praktischen Ausbilder erwarten also „PerF Plus“-Teilnehmer, die über ein B1-Niveau im Deutschen gemäß dem „Gemeinsamen Europäischen Referenzrahmen für Sprachen" (GER) verfügen. ${ }^{11}$ Darauf aufbauend sollen dann während der Maßnahme parallel zum praktischen Unterricht in 365 Zeitstunden berufsbezogene Deutschkenntnisse vermittelt werden. Die Realität der von uns begleiteten Maßnahme sah aber ganz anders aus: Aufgrund des Mangels an Integrationskur-

sen auf dem Land hatte keiner der Teilnehmenden einen Deutschkurs besucht. Über die Gründe, warum man dennoch Flüchtlinge ohne Deutschkenntnisse in den Maßnahmen aufnimmt, berichtete uns der Leiter des Trägers zu Beginn des Kurses Folgendes: ${ }^{12}$

Die PerF-Maßnahme ist aa wichtig, weil's oft einfach äh äh, wenn die Flüchtlinge gekommen sind oder kommen, dann fallens zu erste Mal ins Loch, weil es passiert nichts bis Integrationskursgenehmigung kummt, und wo ma a halt gschaut hat, grad von der Agentur für Arbeit, Mensch, wir wollen euch aufnehmen, wir wollen schaun, dass glei was passiert, weil es ist schon so, die ersten Erfahrungswerte aa von uns, wenn Flüchtlinge einfach wochenlang zuhause sitzen und es passiert nichts, dann verlierens Motivation und depressiv und und und, äh wo es dann ehrlich zu erstma Monate brauchst, bist du das wieder aufbaust. (Interview vom 27.6.2016, 8:40-9:25)

Da die Flüchtlinge in ländlichen Regionen zum Teil sehr lange auf einen Sprachkurs warten müssen, scheint es sinnvoll, sie früher in berufliche Maßnahmen zu integrieren, damit sie dort bereits Deutsch lernen können. Wir konnten jedoch beobachten, dass die Ausbilder mit der Situation, nur Sprachlerner mit fast keinen Deutschkompetenzen vor sich zu haben, überfordert waren. So waren zu Beginn der Maßnahme aufgrund der sprachlichen Verständigungsschwierigkeiten „Standortbestimmung“, „Bedarfsanalyse“ und „Eignungsfeststellung" nicht möglich. In den folgenden Interaktionsanalysen untersuchen wir typische Situationen, die sich aus dieser Problemlage in der praktischen Ausbildung in den Holz- und Metallwerkstätten ergeben haben.

\subsubsection{Interaktionsanalysen}

Als erstes Beispiel analysieren wir einen Ausschnitt aus einer Instruktion, die die aus fehlenden Deutschkenntnissen entstehenden Verständigungsprobleme und die Schwierigkeiten der Ausbilder, mit den sprachlichen Defiziten der Flüchtlinge umzugehen, verdeutlicht. Es handelt sich um ein Beispiel aus der Metallwerkstatt. Die Teilnehmer hatten,

den uns vom Träger vorgegeben. Videoaufnahmen im Krankenhaus durften wir aus Patientenschutzgründen nicht machen.

10 Infoblatt der Arbeitsagentur (2017) zur Maßnahme „Perspektiven für Flüchtlinge“.

11 Der GER unterscheidet sechs Niveaustufen: A1 (Anfänger), A2 (grundlegende Kenntnisse), B1 (fortgeschrittene Sprachverwendung), B2 (selbständige Sprachverwendung), C1 (fachkundige Sprachkenntnisse) und C2 (annähernd erstsprachliche Kenntnisse). Siehe auch online unter: www.europaeischerreferenzrahmen.de (Stand: 23.6.2018).

12 Nur ein Teilnehmer beherrschte aufgrund von Selbststudien Deutsch auf A2/B1-Niveau. 
angeleitet vom Ausbilder, einen Fahrradständer zu bauen. In \#1 erklärt der Ausbilder (WS) zwei syrischen Praktikanten (IH und MM), wie eine Säulen- bzw. Tischbohrmaschine zu bedienen ist.

\#1 Transkript „keine Handschuhe“ („PerF Plus“, Metall, 8.8.16, arb6, 5:08-5:45)

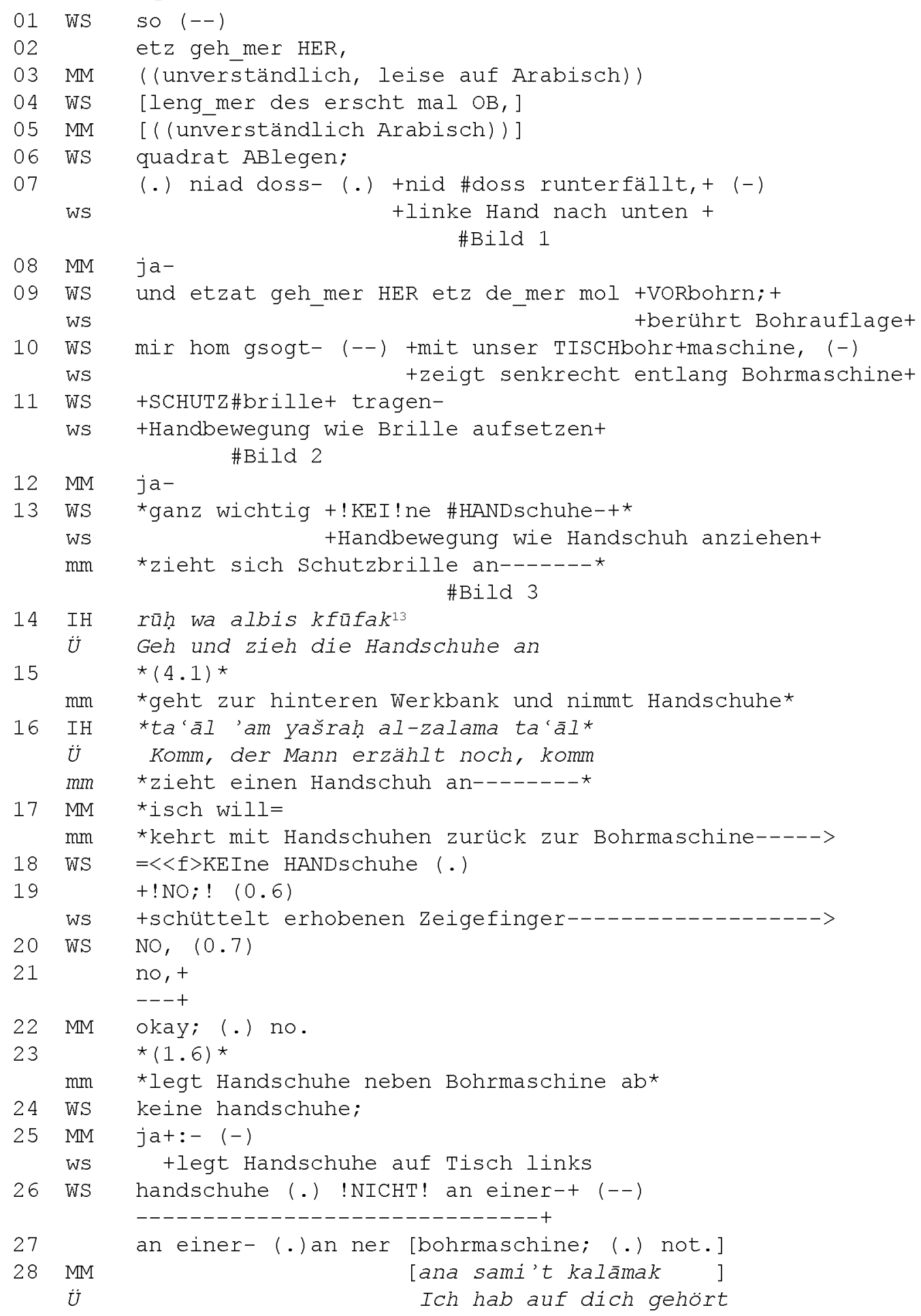

13 Das Arabische steht in den Transkripten kursiv. Wir haben uns bei der Transkription für eine Transliteration entschieden, weil insbesondere bei Parallelpassagen die Schreibrichtung des Arabischen von rechts nach links zu Unklarheiten führen würde. 
Der Ausbilder (WS, links in Bild 1) geht mit den beiden Praktikanten MM (rechts in Bild 1) und IH (auf Bild 1 in der Mitte leicht verdeckt) an eine Tischbohrmaschine und erklärt ihnen, was bei Metallbohrungen zu beachten ist. Dabei legt er die Metallplatte, in die gebohrt werden soll, zunächst zur Seite und kommentiert: „etz geh_mer HER, leng_mer des erscht mal OB, quadrat ABlegen; niad doss- (.) nid doss runterfällt," (02-07). Seine Äußerung illustrierend bewegt er seine linke Hand (Pfeil in Bild 1) von der Metallplatte, die links neben der Bohrmaschine auf dem Tisch liegt, zunächst nach oben und dann nach links unten abfallend.

Im Anschluss geht der Ausbilder auf zwei Sicherheitsaspekte ein, die man bei der Arbeit mit Tischbohrmaschinen beachten muss: die Verwendung einer Schutzbrille und den Verzicht auf Arbeitshandschuhe. Bei der Instruktion ,mir hom gsogt- (--) mit unser TISCHbohrmaschine, (-) SCHUTZbrille tragen-" (10-11) macht WS eine Bewegung mit beiden Händen, als ob er sich eine Brille aufsetzen würde (Bild 2).

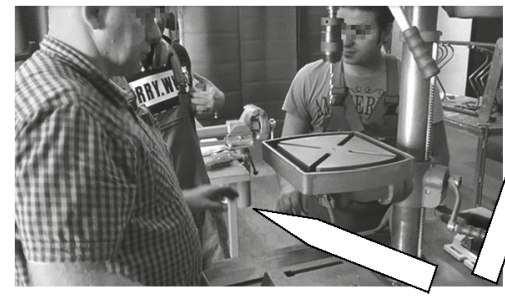

Bild 1: „nid doss ${ }^{14}$ runterfällt,“ (07)

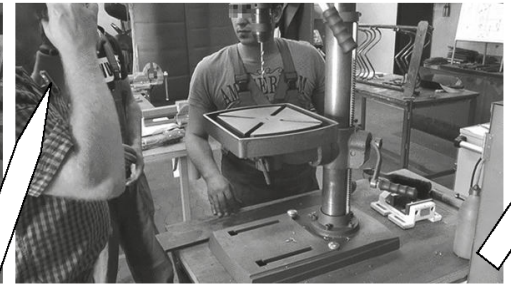

Bild 2: „SCHUTZbrille tragen-“ (11)

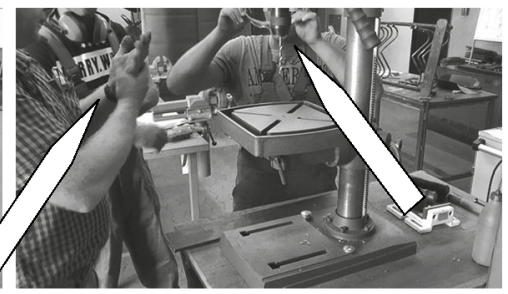

Bild 3: „!KEI!ne HANDschuhe-“ (13)

MM reagiert mit einem Verstehen anzeigenden ,ja“ (12). IH kommt der Aufforderung nach und setzt seine Schutzbrille, die er in die Haare gesteckt hatte, auf (Bild 3, rechts). Als nächste wichtige Sicherheitsmaßnahme erwähnt der Ausbilder, dass an der Bohrmaschine keine Handschuhe zu tragen sind: ,ganz wichtig !KEI!ne HANDschuhe-“ (13). Der Grund für diese Sicherheitsvorkehrung liegt darin, dass überall dort, wo man es mit rotierenden Teilen zu tun hat, die Gefahr des Aufwickelns und schwerer Fingerverletzungen besteht. Auch diesmal begleitet der Ausbilder seine Äußerung gestisch: Er hebt beide Hände auf Brusthöhe und bewegt seine rechte Hand so, als ob er sich an die linke Hand einen Handschuh anzöge (Bild 3, links).

Durch die Parallelität dreier aufeinander folgender Instruktionen und jeweiliger gestischer Illustration bekommt die Sequenz einen Listencharakter. Dies verstärkt das Potenzial der Handschuh-Anzieh-Gestik, zu einem Missverständnis zu führen: Der Ausbilder vermittelt gestisch (Handschuhe anziehen) das Gegenteil dessen, was er verbal mitteilt (keine Handschuhe anziehen). In einer Kommunikationssituation mit Muttersprachlern würde diese Diskrepanz aufgrund des Verständnisses des Gesagten wohl kaum zu einem Missverständnis führen. In der vorliegenden Interaktion dagegen orientieren sich die Adressaten an der Geste und verstehen diese irrtümlich als positive Instruktion. Der ältere Praktikant IH wendet sich dem jüngeren zu und fordert ihn auf Arabisch auf: „rūḥ wa albis kfüfak“ (Geh und zieh die Handschuhe an, 14). „Handschuhe" hat IH wohl verstanden, zumal diese in der Metallwerkstatt ständig thematisiert werden. Aber schon die Negation „keine" kann Probleme bereiten. Durch die Lautähnlichkeit von „keine“ und „deine“ kann der Praktikant die Äußerung auch als „ganz wichtig deine Handschuhe“ verstanden haben, was ja

14 Fettgedruckte Silben markieren den Moment, an dem der Screenshot gezogen wurde. 
bei der zu erwartenden Kongruenz von Äußerung und redebegleitender Geste passen würde. In dem Fall wäre es kein Nicht-Verstehen, sondern ein Missverstehen. Die Trennlinie zwischen Miss- und Nicht-Verstehen ist aber hier wie auch in vielen anderen Fällen in unseren Daten nicht immer eindeutig. ${ }^{15}$

Der jüngere Praktikant MM kommt der Aufforderung nach und geht nach hinten zur Werkbank, wo sich die Handschuhe befinden. Da aber IH bemerkt, dass der Ausbilder etwas verwundert und mit halb offenem Mund das Geschehen verfolgt, fordert er MM auf zurückzukommen: „ta āl 'am yašrah al-zalama ta 'âl" (Komm, der Mann erzählt noch, komm, 16). Jetzt interveniert auch der Ausbilder, der inzwischen realisiert, dass die syrischen Praktikanten seine negierte Äußerung falsch verstanden haben: „, $=<\mathrm{f}>$ KEIne HANDschuhe (.) !NO;! (0.6) NO, (0.7) no, “ (18-21). Mit dem mehrmaligen Wiederholen des sehr lauten No sowie dem Schütteln des erhobenen Zeigefingers hebt WS einerseits die große Bedeutung dieser Sicherheitsmaßnahme an der Bohrmaschine hervor. Andererseits signalisiert er mit dem Codeswitch ins Englische sein Verständnis, dass das Verstehensproblem in mangelnden Deutschkenntnissen des Praktikanten bestehe. Während dies natürlich grundsätzlich zutreffend ist, ist aber gerade nicht anzunehmen, dass dieser ausgerechnet die deutsche Negationspartikel nein, die zu den ersten zu lernenden Ausdrücken in der Fremdsprache gehört, nicht verstehen kann. Der Codeswitch und seine mehrmalige, laut hervorgehobene Wiederholung sind daher für den Praktikanten gesichtsverletztend (vgl. Goffman 1955), da ihm damit auch die basalsten Deutschkenntnisse abgesprochen werden. ${ }^{16}$ Zudem ist der Codeswitch pragmatisch paradox: Nachdem der Ausbilder zuvor komplexe Satzkonstruktionen und fachsprachliche Komposita benutzte, behandelt er den Flüchtling nunmehr als jemanden, der selbst einfachste Ausdrücke des Deutschen nicht kennt.

In der Folge akkommodiert sich MM dem Codeswitch mit einer expliziten Verstehensbehauptung (,okay; (.) no.“, 22), zieht den Handschuh wieder aus und legt beide Handschuhe neben die Tischbohrmaschine (23). Daraufhin wiederholt der Ausbilder noch einmal seine negierte Instruktion (,keine handschuhe;“" 24), diesmal auf Deutsch. Nun benutzt er die syntaktische Negation „nicht" statt der morphologischen „keine“" und wiederholt seine Aufforderung: „handschuhe (.) !NICHT! an einer- (--) an einer- (.) an ner bohrmaschine; (.) not.“ (26-27). Eine Erklärung dafür gibt er nicht, sondern begleitet seine Äußerung mit einer weiteren tendenziell gesichtsverletzenden Handlung: Er nimmt die Handschuhe und legt sie auf den Tisch, der hinter ihm links steht. Diese Handlung vermittelt, dass der Ausbilder den Praktikanten nicht vertraut und deshalb die Handschuhe aus ihrer Reichweite

15 Hinnenkamp (1998, S. 12) meint: „Mißverstehen und Nichtverstehen sowie Mißverständnisse und Nichtverständnisse sind von unterschiedlicher Art. Mißverstehen und Mißverständnisse haben - zumindest nach einer Art Auffassung - nämlich durchaus ein Verstehen zur Voraussetzung, das sich dann als falsch herausstellt. [...] Mißverständnisse sind demnach falsifizierte Verständnisse." Obwohl dies sicherlich korrekt ist, ist die Unterscheidung doch nicht so eindeutig. Denn Nichtverstehen kann zu einer Hypothesenbildung führen, die dann einem Missverstehen gleichkommt. In Bezug auf ihre sprachliche Dokumentation unterscheiden sich Nichtverstehen und Missverstehen dagegen deutlich. Während Nichtverstehen als solches vom Nichtverstehenden direkt angezeigt wird, kann ein Missverständnis immer erst im Nachhinein von Handlungen, die Verstehen beanspruchen bzw. auf der Basis eines Verständnisses ausgeführt werden, angezeigt werden - entweder vom Produzenten des Verstehensgegenstands selbst oder vom Missverstehenden anhand des Gewahrwerdens von Konsequenzen, die nicht zum Verstandenen passen.

16 Der Codeswitch in ein basales Englisch kann deshalb als eine Variante des foreigner-talk angesehen werden (vgl. Ferguson 1975; Hinnenkamp 1982). 
entfernt. Anstelle einer Erklärung, die auf die Einsicht des Adressaten setzen würde, wird in paternalisierender Weise das Setting so arrangiert, dass potenzielle Gefahrenquellen aus der unmittelbaren Umgebung des Auszubildenden entfernt werden. Damit wird ihm abgesprochen, selbstverantwortlich und aufgrund seines Verständnisses der Sicherheitserfordernisse der Aufgabe situationsangemessen zu handeln. Die Sequenz wird damit beendet, dass der jüngere Praktikant MM seinem älteren Kollegen IH auf Arabisch implizit die Schuld für sein Fehlverhalten vorwirft, da er nur das gemacht habe, was dieser ihm aufgetragen hat: ,ana sami't kalāmak“" (Ich hab auf dich gehört, 28).

Das zweite Beispiel stammt ebenfalls aus der Metallwerkstatt. Diesmal ist der Ausbilder WS (rechts auf Bild 4) allein mit dem Praktikanten IH (links auf Bild 4). Sie sind mit der Arbeit an einem Fahrradständer (auf Bild 4 Mitte links) fast fertig. IH hat nur noch die letzten beiden Radhalterungen zusammenzuschweißen. Nachdem er die erste Halterung angeschweißt hat, kontrolliert der Ausbilder die Arbeit. Dabei bricht die Halterung ab (auf Bild 4 hält der Ausbilder sie in der rechten Hand). Die Interaktion in Ausschnitt \#2 folgt unmittelbar danach. Der Ausbilder führt aus, dass solche Fehler, wie nicht gut genug geschweißt zu haben, zu einer Ausbildung gehören und nicht schlimm sind (01-20). Anschließend erklärt er die Ursache des Fehlers (22-42), legt dar, was verbessert werden muss (43-54), und formuliert den Plan für die nächsten Handlungen (55-58), mit denen der Fehler behoben werden soll.

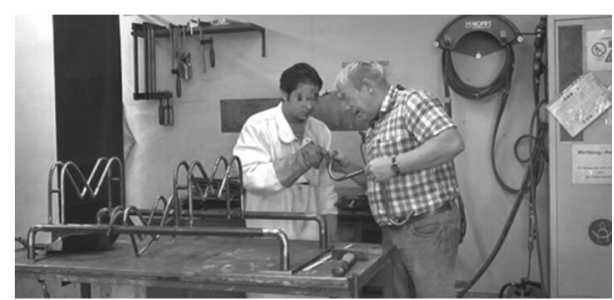

Bild 4: „des sieht ma jetzt a DA:“ (22)

\#2 Transkript „wo keine Späne fallen“ („PerF Plus“, Metall, 5.8.16, 14:34-16:18)

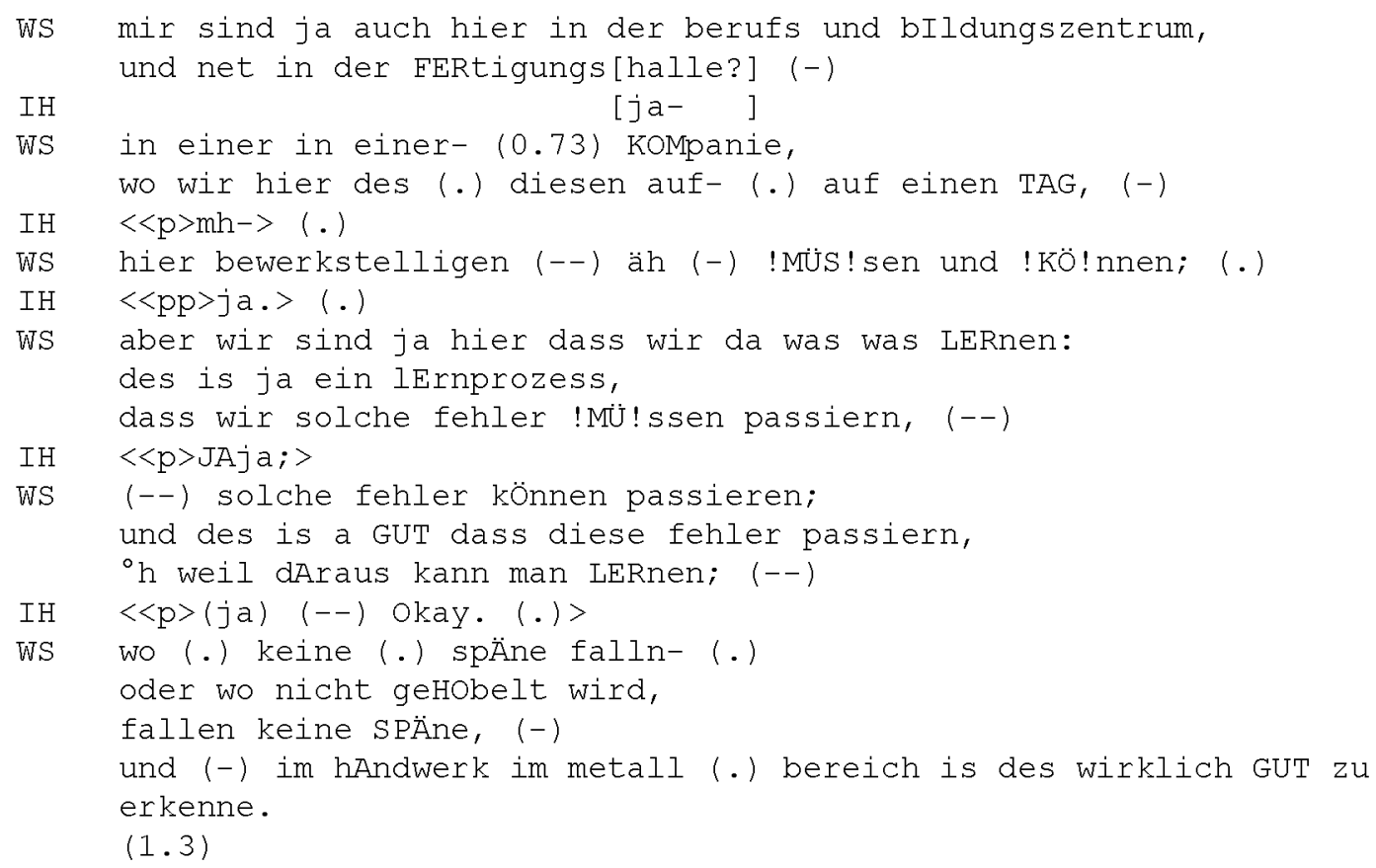


$50 \quad \mathrm{IH}$

so un <<all> des sieht ma jetz a +DA:?> (.)

tzeigt Bruchstelle an

Halterung-->

da hom mir *äh\#*- (.)

*berührt Bruchstelle* \#Bild 4

ja? (--)

eine geFÜgeveränderung gehabt-

zwischen VOLLma+terial und (.) und und äh (--)+PRO[fIl? ]

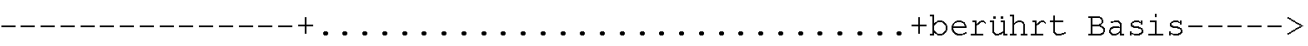

des heißt+ in [des] zollROHR, $\left[\begin{array}{ll}\mathrm{xXX} & \mathrm{XXX}\end{array}\right]$ 


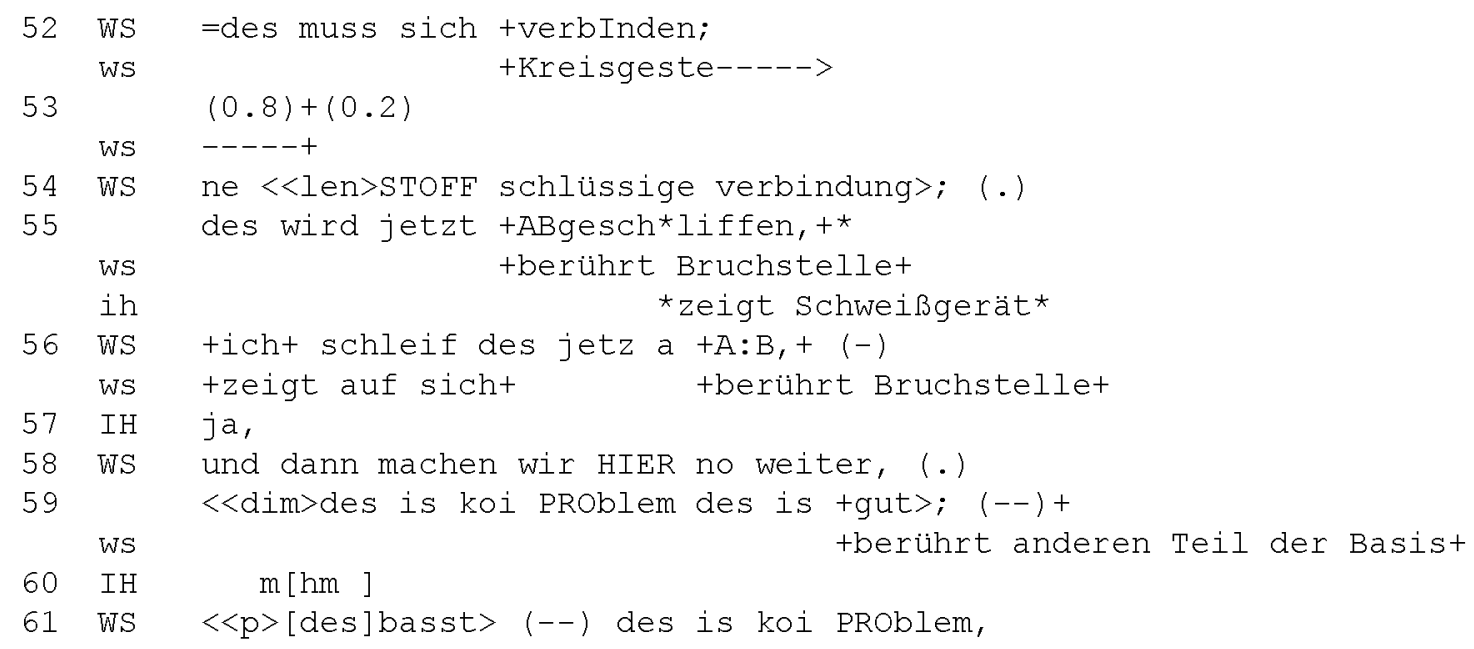

Der Ausbilder WS gibt eine lange Erklärung über Sinn und Notwendigkeit von Fehlern im Lernprozess (01-20). Der Praktikant IH schaut ihn währenddessen kontinuierlich an und produziert zunächst einige leise Rückmeldesignale $(06,08,12,16)$, die Aufmerksamkeit anzeigen. Es bleibt aber ungewiss, ob und in welchen Aspekten er den langen, syntaktisch komplexen und durch zahlreiche Selbstreformulierungen charakterisierten Turn des Ausbilders versteht. ${ }^{17}$ Auf das diesen Teil seines Beitrags abschließende, modifizierte figurative Sprichwort („oder wo nicht geHObelt wird, fallen keine SPÄne, $\left.(-)^{“}, 18-19\right)$ reagiert IH nicht. In diesem Abschnitt spricht WS über Abstraktes; allein die Sprache trägt die Last der Kommunikation. WS versucht nicht, IHs Verstehen zu prüfen. Seine Selbstreformulierungen und Elaborierungen mögen durch den Versuch, sich IH besser verständlich zu machen, motiviert sein, sind aber nicht erkennbar erfolgreich und tragen eher dazu bei, den Beitrag schwieriger zu machen (z.B. Kontrast mit der „FERtigungshalle?", 02). Dass WS seine Äußerung an den spezifischen Adressaten anzupassen versucht, wird hier am deutlichsten in der Verwendung des Ausdrucks „KOMpanie“ (04), offenbar einer nicht usuellen Entlehnung des englischen Ausdrucks company. Ähnlich wie in Beispiel 1 rekurriert WS zur Verständigungserleichterung auf das Englische, allerdings wiederum in einer weder an die Kenntnisse des Adressaten noch die vorliegende Darstellungsaufgabe angepasste Weise.

In der anschließenden Erklärung der Ursache für das Misslingen des Anschweißens nutzt WS dagegen die Möglichkeit, durch gestische Demonstrationen an den kritischen Objekten Referenzen und ansatzweise auch Handlungen zu verdeutlichen (22-40). ${ }^{18}$ So gelingt zumindest die erkennbare Herstellung von common ground (vgl. Clark 1992) hinsichtlich der jeweils thematisierten Gegenstände (22: Zeigen auf Bruchstelle an der Halterung; 32, 46/49: Berührung der Stelle am Basisträger, an der die Halterung angeschweißt wurde). IH zeigt jeweils unmittelbar auf WS' Gesten folgend durch eigene korrespondierende Zeigegesten bzw. Berührungen an den betreffenden Stellen der Objekte an, dass er verstanden hat, worauf sich WS bezieht (23: Bild 4; 32: Bild 5; 49: Bild 6). ${ }^{19}$

$17 \mathrm{Zu}$ Selbstreformulierungen siehe Gülich/Kotschi (1996).

18 Zum kommunikativen Umgang der Ausbilder mit eigenen Fehlern in der betrieblichen Ausbildung siehe auch Brünner (1987, S. 266-295)

19 Die Wiederholung von redebegleitenden Gesten durch den Rezipienten ist eine gängige Praktik der Anzeige von Verstehen in der Interaktion, siehe z.B. Mol et al. (2012) und Yasui (2013). 


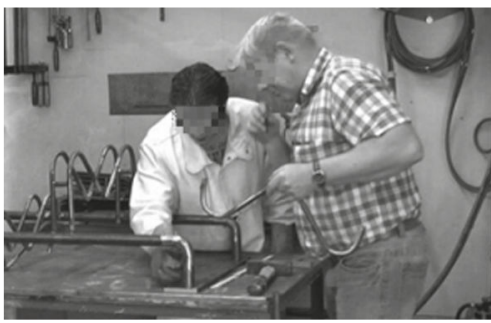

Bild 5: ,die wÄrme ent wicklung," (32)

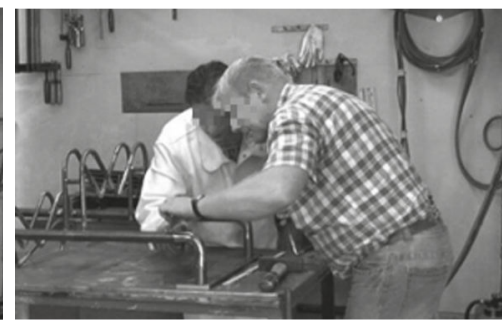

Bild 6: , ja-(.) dass wir da $\operatorname{dieses}^{c}(48)$

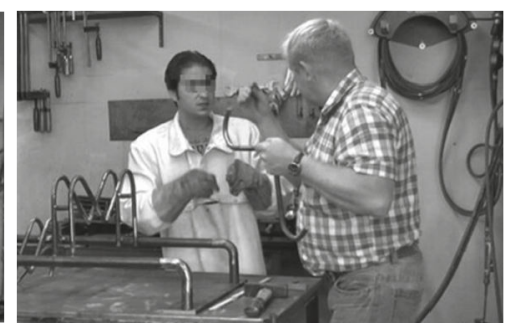

Bild 7: ,ja des is äh:: (-)“ (40)

WS benutzt ikonische Gesten zur Verdeutlichung, dass die Materialien richtig verschmelzen müssen - auch diese Geste wiederholt IH ansatzweise, allerdings deutlich zaghafter und unvollständiger (38-41, Bild 7).

IHs Geste scheint hier anzuzeigen, dass ihm deutlich geworden ist, dass die Materialien zusammengebracht werden müssen, die Spezifik der Art des Zusammenbringens (die Verschmelzung) drücken sie nicht aus. In diesem Abschnitt seiner Instruktion beobachtet WS wiederholt die Reaktionen von IH. So wiederholt er seine zentrale Kategorisierung „verschmelzen" und die begleitende illustrative Geste, als er IH's gestisch unsicheren und im verbalen Teil abgebrochenen Verstehensdokumentationsversuch wahrnimmt (40-41). Mehrfach markiert er Aufmerksamkeitsrelevantes durch die Rückversicherungspartikel ,ja, “ $(24,44,50)$. Während elementare Referenzsicherungen hier gelingen, zeigt nicht nur IHs unsichere verbal-gestische Reaktion in 40, dass es sehr unwahrscheinlich ist, dass er Wesentliches von WS' Ursachenerklärung verstanden hat. WS benutzt eine Fülle von Fachausdrücken (wie Gefügeveränderung, Vollmaterial, Zollrohr, Wärmeentwicklung u.a.), die IH unbekannt sein dürften und auch nicht weiter erläutert werden.

In der anschließenden Planung der nächsten Handlungsschritte zur Fehlerbehebung verdeutlicht WS nochmals das Ziel, dass sich die Stoffe verschmelzen müssen. Er reformuliert den bezweckten Vorgang dreimal in 48-54 und bezeichnet ihn schließlich mit dem fachlich gültigen Ausdruck „,<len $>$ STOFF schlüssige verbindung $>$;“ (54). Mit noch expressiveren Kreisgesten, die jeweils mit der Verbalisierung des Vorgangs koordiniert sind, versucht er den Vorgang zu veranschaulichen (48, Bild 8), IH reagiert zunächst mit einer kleineren und nur den halben Radius umfassenden Drehgeste (50, Bild 9). Weitere Verstehensdokumentationen zeigt er nicht.

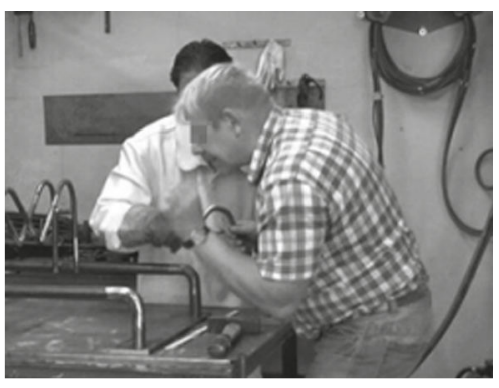

Bild 8: „dass des REINbrennt;“ (48)

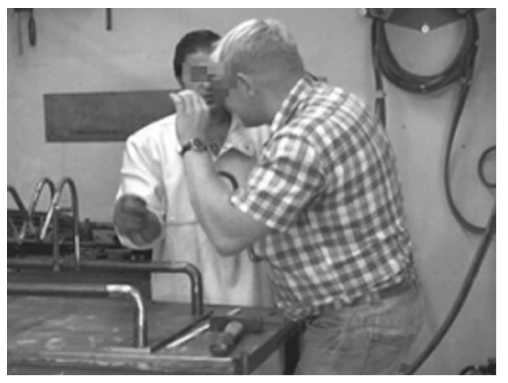

Bild 9: ,ja-“ (50)

Als WS den Fokus auf die nächste vorzunehmende Handlung (,des wird jetzt ABgeschliffen", 55) verschiebt, zeigt IH auf das Schweißgerät. Er versteht also offenbar, dass WS nun zur Instruktion des nächsten Handlungsschritts übergegangen ist, nicht aber, welcher 
Handlungsschritt de facto angesprochen wurde. Vielmehr scheint die situativ konstituierte Erwartung, dass die abgebrochene Halterung wieder angeschweißt werden muss, die Grundlage für sein Verständnis des nächsten anstehenden Schritts zu sein. WS reagiert dagegen mit einer verdeutlichenden Selbstreformulierung, indem er auf sich selbst zeigt und erklärt, dass er selbst nun abschleife (56).

Zusammenfassend zeigt sich in diesem Ausschnitt, dass trotz permanenter intensiver Aufmerksamkeit von IH auf WS' Instruktion wechselseitige Verständigung zweifelsfrei nur hinsichtlich elementarer Objektreferenzen (die Stellen, an denen die verschweißten Teile auseinandergebrochen sind) mithilfe referenzieller Gesten und hinsichtlich der globalen Handlungsstruktur von WS' Turn (Übergang zur Ursachenerklärung, Übergang zur Ankündigung nächster Schritte) gelingt. Die abstrakteren Darstellungen zu Beginn, die genauen fachlichen Erklärungen sowie schließlich die Ankündigung konkreter nächster Handlungen dagegen werden nicht erkennbar verstanden. WS scheinen die Verstehensprobleme an einigen Stellen bewusst zu sein. Verstehensabsicherungen, die die Beobachtung des Adressaten involvieren, betreibt er nur hinsichtlich der Referenzklärung. Ansonsten begegnet er teilweise durch Selbstreformulierungen, gestische Demonstrationen sowie langsameres Aussprechen zentraler Ausdrücke $(38,41,43,54)$ den Verstehensproblemen des Adressaten.

Die nächsten beiden Interaktionsbeispiele stammen aus der Holzwerkstatt, die auf den praktischen Unterricht in der Metallwerkstatt folgte. Bei dieser Arbeit verfolgt der Ausbilder GM das Ziel, innerhalb der Praktikumszeit mit den Flüchtlingen ein Gartenhäuschen aus Holz zu bauen. Im Transkriptausschnitt 3 fordert er die beiden Praktikanten (PL und DS) auf, einen breiten Stechbeitel und einen Holzhammer zu holen (01).

\#3 Transkript „stechbeitel und holzhammer“ („PerF Plus“, Holz, 17.10.16, 00003 , $5: 08-5: 39)$

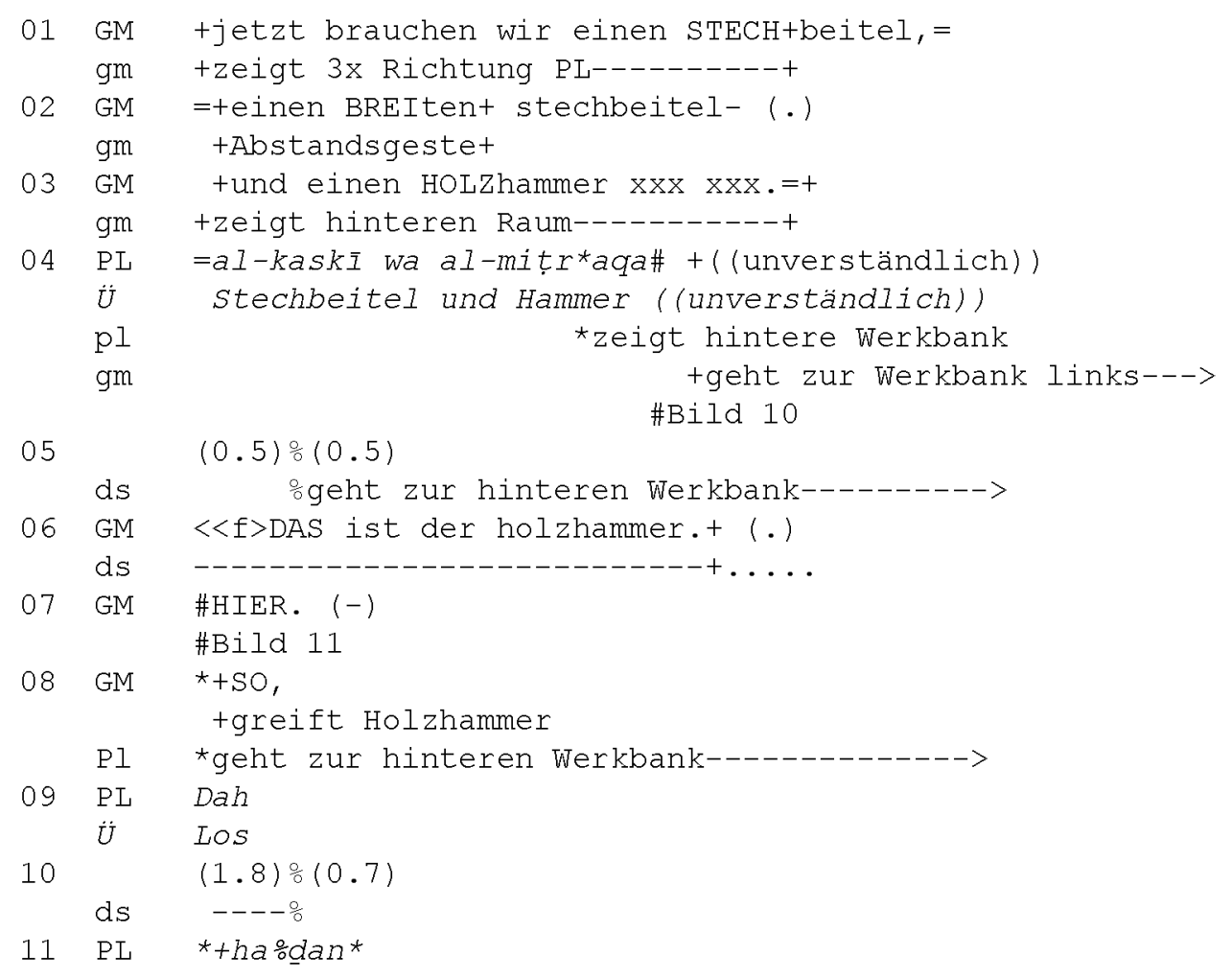




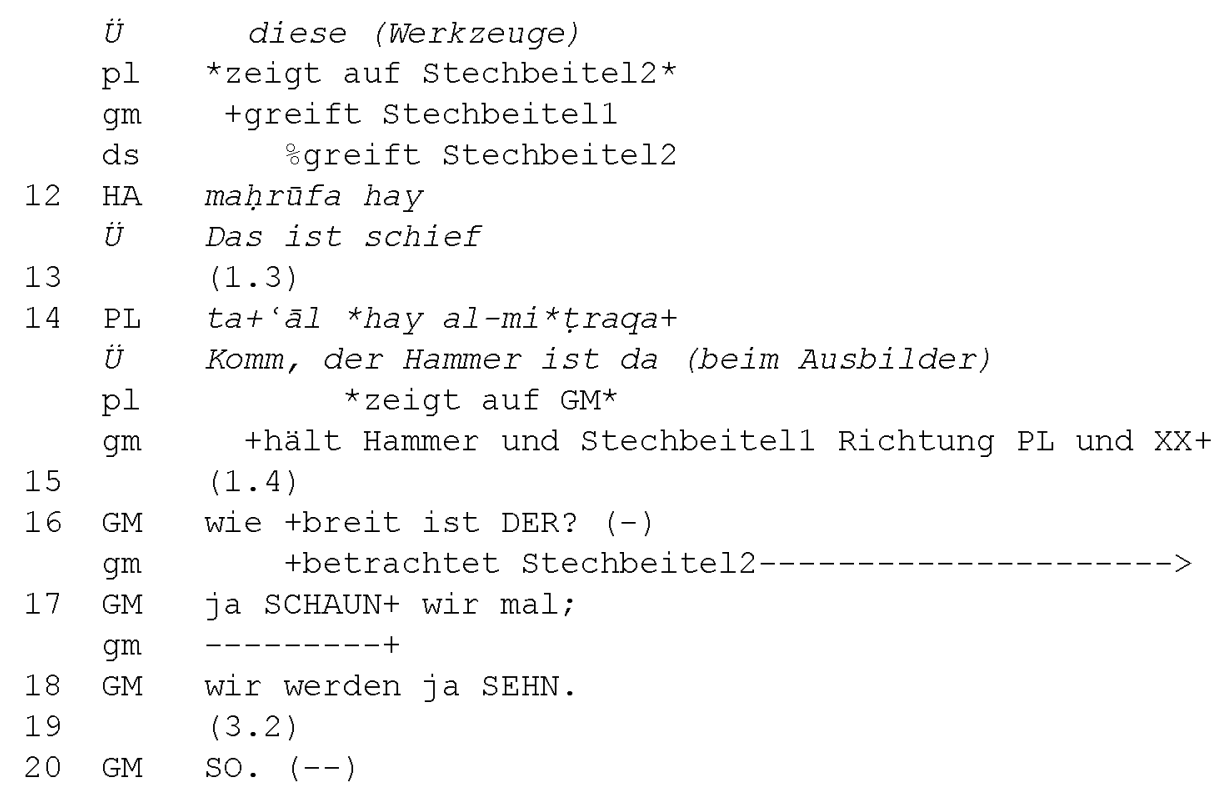

PL, der einzige Kursteilnehmer mit Deutschkenntnissen auf A2/B1-Niveau, übersetzt für DS die Aufforderung GMs, Stechbeitel und Holzhammer zu holen (01-03), ins Arabische (04, Bild 10). PL agiert in diesem Ausschnitt wie auch sonst als Dolmetscher vom Deutschen ins Arabische, definiert sich aber selbst nicht als primären Adressaten der Instruktionen, denn er führt selbst die instruierten Handlungen nicht aus. PL zeigt während seiner Dolmetschung zur hinteren Werkbank (04), wohin auch GM bei seiner Instruktion gezeigt hatte (03). Während DS zu dieser Werkbank geht (04-10), um die Arbeitsgeräte zu holen, wendet sich der Ausbilder einer anderen Werkbank links von ihnen zu (04-06) und holt dort selbst die entsprechenden Werkzeuge $(08,11$; Bild 11). So kommt es, dass DS und GM nahezu zeitgleich unabhängig voneinander einen Stechbeitel ergreifen (11). Als PL entdeckt, dass GM bereits einen Holzhammer gefunden hat, während DS auf der hinteren Werkbank keinen finden konnte, macht er DS darauf aufmerksam (14). Gleichzeitig präsentiert GM DS die Werkzeuge, die er besorgt hat (14), und betrachtet dann den von DS besorgten Stechbeitel (16-17), den er offenbar für zu schmal hält (vgl. GMs Instruktion in 02).

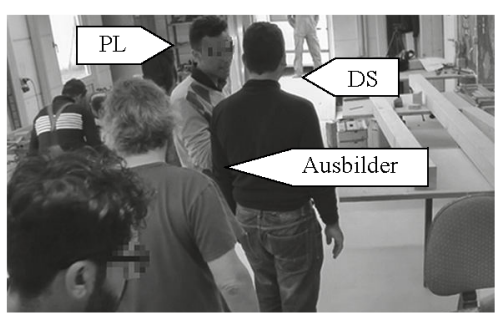

Bild 10: ,=al-kaskīwa al-miṭr*aqa\# $+((\text { unverständlich }))^{\text {c }}$

Ü: Stechbeitel und Hammer ((unverständlich)), (04)

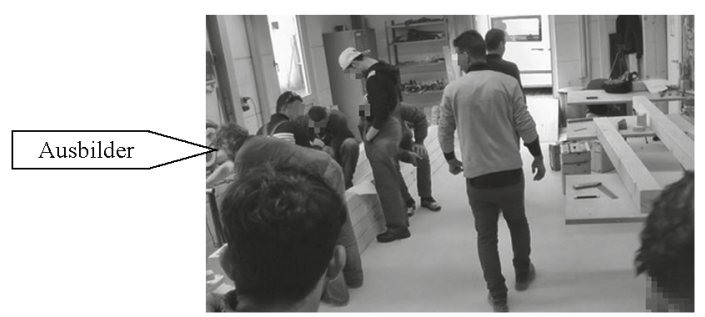

Bild 11: , $<<>$ DAS ist der holzhammer. $+>$ HIER," (06-07)

Die Interaktion zwischen GM und DS wird im Ausschnitt vollständig von PL als Dolmetscher vermittelt. DS zeigt in keiner Weise an, dass er GMs Instruktionen unabhängig von der Dolmetschung versteht, er reagiert auch nicht auf GMs Frage und Kommentar zu dem von DS besorgten Stechbeitel (16-18). GM hingegen trägt in seinem Interaktionsverhalten 
in keiner Weise der Tatsache Rechnung, dass die Verständigung mit DS ausschließlich über die (zutreffenden) Dolmetschungen PLs gesichert werden kann. Er adressiert mit Blick und Körperhaltung ausschließlich DS, wendet sich an keiner Stelle PL zu und wartet mit nächsten Interaktionsbeiträgen bzw. Turnfortsetzungen auch nicht, bis PL gedolmetscht hat. Es ist nicht erkennbar, ob GM überhaupt registriert, dass PL als Dolmetscher agiert. Damit verpasst er es, die in der Situation verfügbaren mehrsprachigen Ressourcen und Kompetenzen systematisch zu nutzen.

Obwohl GM seine Instruktion an DS adressiert (01-03), wartet er nicht, ob es DS gelingt, die gewünschten Arbeitsmaterialien korrekt zu identifizieren und zu holen. Stattdessen erledigt er die Aufgabe selbst. Als DS zumindest einen der beauftragten Gegenstände bringt, signalisiert er weder, dass grundsätzlich das richtige Objekt (Stechbeitel) beschafft wurde, noch macht er explizit, warum er ihn für eventuell nicht geeignet hält. Aus seiner Frage und seinen Kommentaren (16-18) lässt sich nur mit einem bereits bestehenden Hintergrundwissen über die Erfordernisse der anstehenden Holzbearbeitungsaufgabe erschließen, worin das Problem (zu geringe Breite des Stechbeitels, den DS geholt hat) besteht. Dieses Wissen fehlt den Auszubildenden jedoch. In diesem Abschnitt wie auch in anderen ist erkennbar, dass der Ausbilder primär das Ziel verfolgt, das Produkt, das Holzhaus, fertig zu stellen. Er führt (wie hier bei der Beschaffung der Werkzeuge) viele Arbeitsschritte selbst aus und kommentiert sie in einer oft recht impliziten Weise, mit der keine explizite und grundständige Wissensvermittlung erfolgen kann. Die Kursteilnehmer erhalten oft nicht die Gelegenheit ihre Fertigkeiten zu erproben bzw. unter Beweis zu stellen. Es erfolgt häufig keine gezielte Rückmeldung auf ihre Handlungen, insbesondere nicht in positiver Hinsicht.

Im zweiten Beispiel aus der Holzwerkstatt arbeitet der Ausbilder allein mit dem Praktikanten HD. Sie messen einen Balken ab, um die Länge der beiden Zapfen zu bestimmen, die an den Endstücken des Balkens entstehen sollen. Der gesamte Balken ist unbearbeitet $190,5 \mathrm{~cm}$ lang. Nach der Bearbeitung soll er nur noch $179 \mathrm{~cm}$ lang sein, was bedeutet, dass die beiden Zapfen an den Enden insgesamt $11,5 \mathrm{~cm}$, also z.B. jeweils $6 \mathrm{~cm}$ und $5,5 \mathrm{~cm}$, lang sein können.

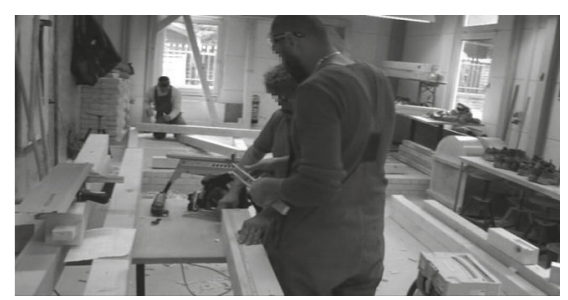

Bild 12: „fünf komma FüNF,“ (08)

\#4 Transkript „oder fünf komma sieben is egal“ („PerF Plus“, Holz, 19.10.16, 00011, $0: 03-1: 27)$

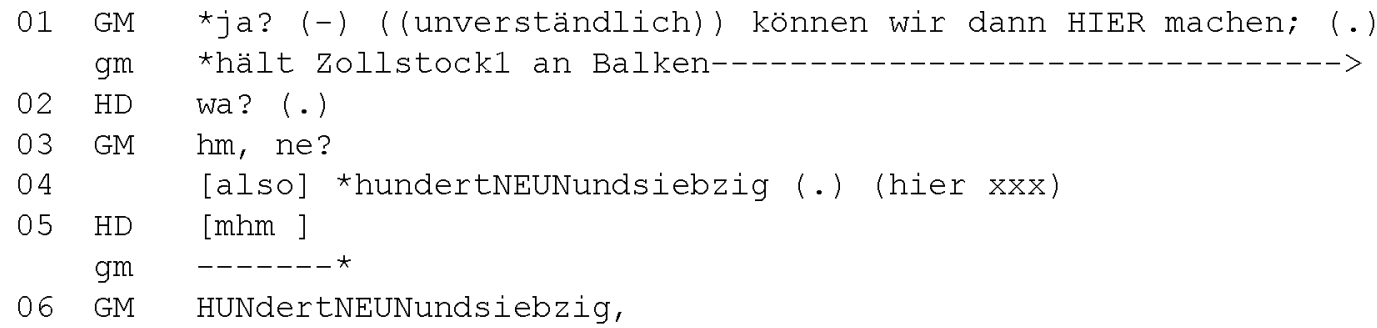




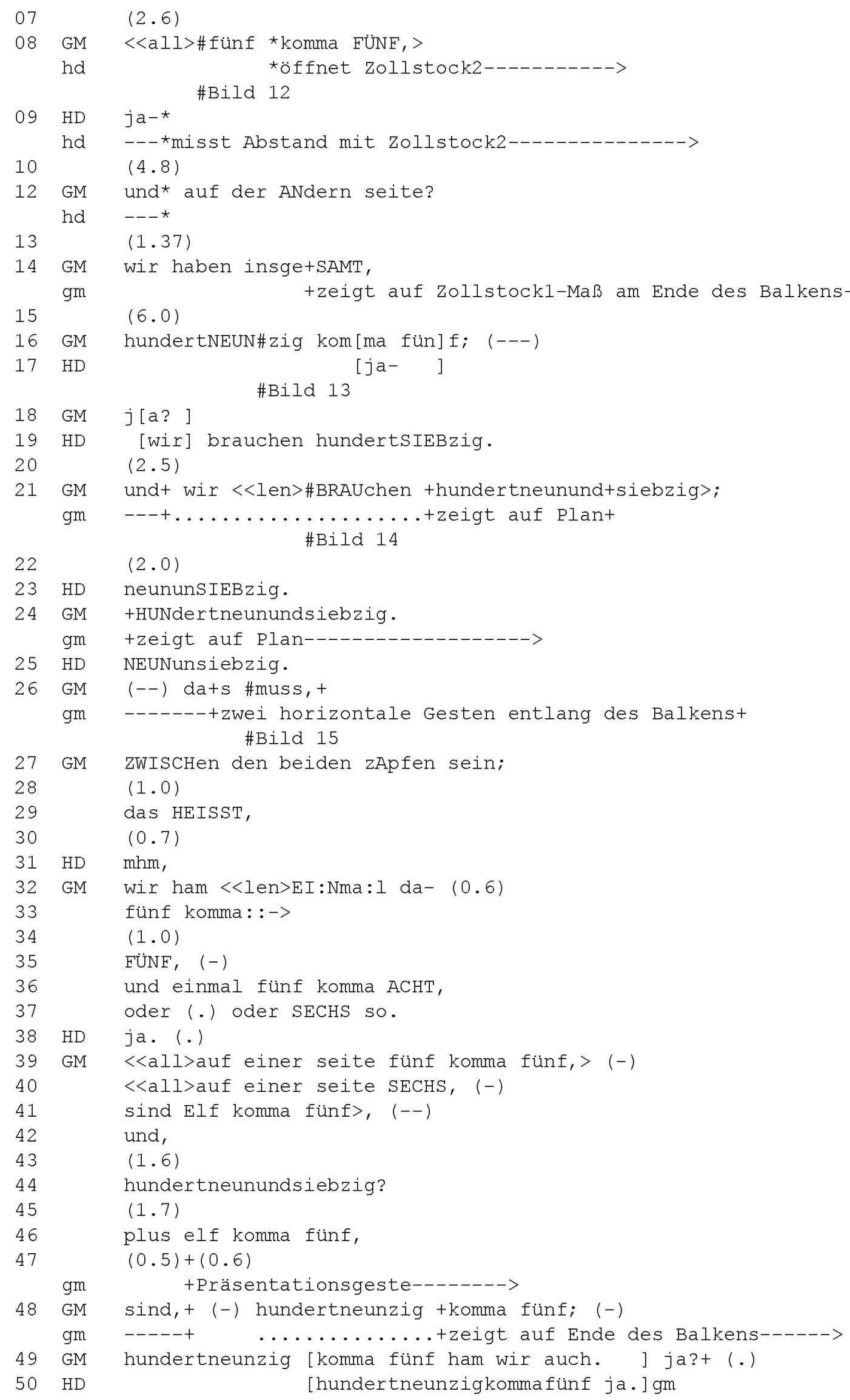




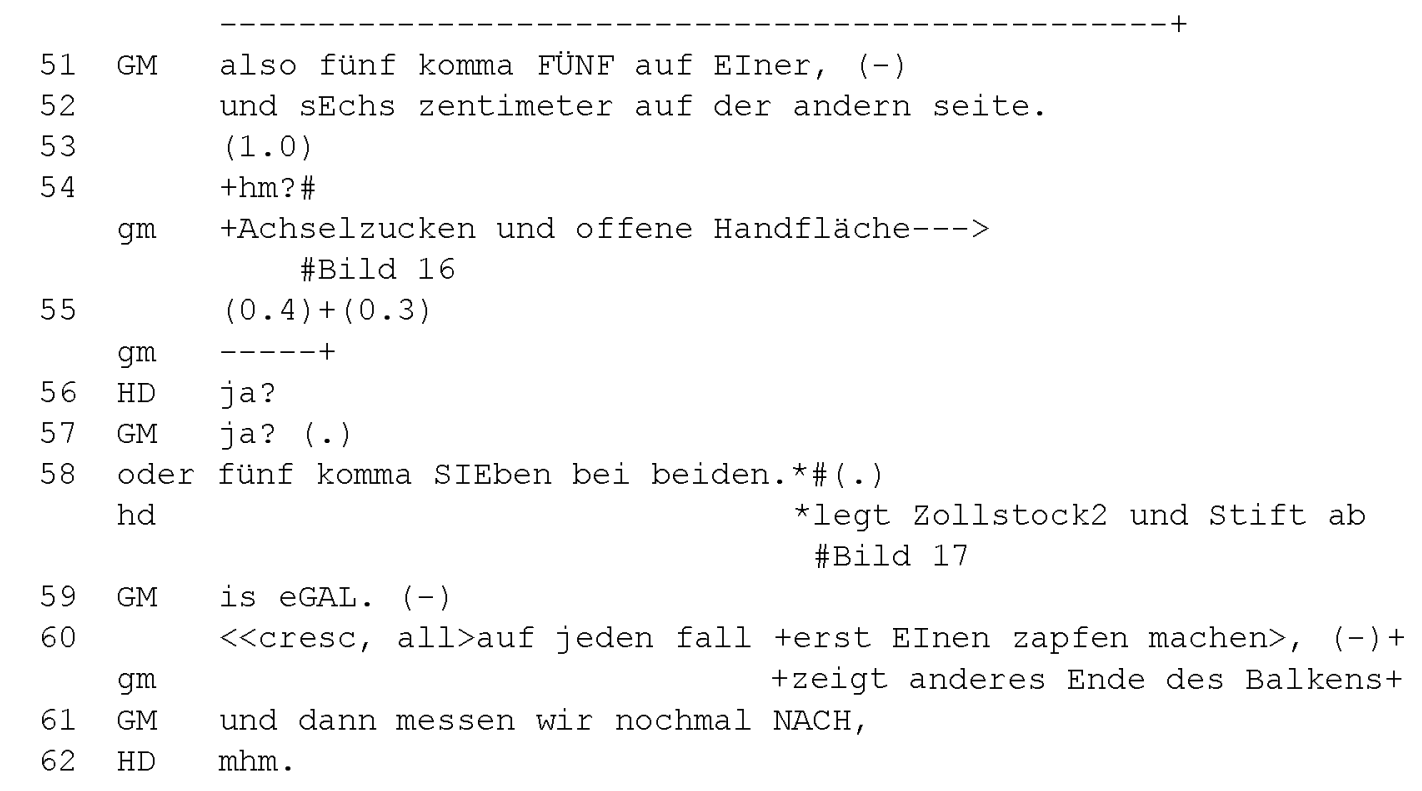

Die Aufgabe, Gegenstände (hier Holz) abzumessen und aufgrund von Berechnungen zuzuschneiden, ist im Handwerk von grundlegender Bedeutung. Daher ist es wichtig, dass die Praktikanten Zahlen, Maßeinheiten ( $\mathrm{cm}, \mathrm{mm}$ etc.) und Rechenausdrücke (plus, minus etc.) zu beherrschen lernen. Der Ausbilder agiert auf der Basis seines professionellen Wissens, das er jedoch kaum in Form von Instruktionen und Erklärungen explizit macht. Er nennt die für die konkrete Messung relevanten Zahlen (welche auch für ihn selbst fallspezifisch neue Informationen sind), expliziert jedoch nur vereinzelt und dann auch meist in sehr verkürzter Form, welchen Status diese innerhalb der zu unternehmenden Konstruktion haben.

Zunächst nennt er die Zahl 179 (04, 06), ohne zu verdeutlichen, dass dies die Länge ist, die der Balken zwischen den Zapfen haben soll. Sodann nennt er die Zahl 5,5 (08). Bereits zu Beginn der Zahlnennung öffnet der Praktikant HD seinen Zollstock (08, Bild 12) und zeigt damit, dass er verstanden hat, dass etwas abzumessen ist, und misst dann auch den entsprechenden Abstand am einen Ende des Balkens. Aufgrund der räumlich-visuellen Vororientierung von GM und HD auf die Länge des Balkens und die Aufgabe, dass dieser zugeschnitten werden muss, kann HD somit die Zahlennennung GMs korrekt als Instruktion, die entsprechende Länge am einen Ende des Balkens abzumessen, verstehen. Erst nach und nach wird der Ausbilder expliziter in Bezug auf den Status der einzelnen Längenmaße für die Konstruktionsaufgabe. In 14-21 verdeutlicht er ansatzweise die Konstruktionsaufgabe durch die Integration der Messwerte 190,5 und 179 in jeweils ein volles Syntagma ,wir haben insgeSAMT,“ (14) vs. „wir brauchen“ (19), welches den Kontrast von Ist und Soll der Balkenlänge ohne Zapfen darstellt. Dabei produziert GM die Angabe des Sollwerts als Korrektur eines nicht zutreffenden Werts, den HD genannt hatte, nämlich 170 (19). Wiewohl dieser Wert falsch ist, zeigt HD damit doch, dass er die grundsätzliche Handlungsaufgabe verstanden hat, nämlich den Balken zu kürzen.

Während GM verbal kaum den Status der von ihm genannten Zahlen im Rahmen der übergreifenden Handlungsaufgabe expliziert, verdeutlicht er gestisch den gegenständlichen Bezug der Messwerte: 190,5 steht für die Länge des Balkens (er zeigt auf die entsprechende Maßeinheit des Zollstocks am Ende des Balkens, 14-21, Bild 13, sowie 48-50), 179 steht für die durch den Konstruktionsplan definierte Zielgröße. GM zeigt dazu auf den 
Plan (21, Bild 14, sowie in 24) und deutet durch kursorische horizontale Gesten die erforderliche Länge des Balkens an (26, Bild 15).

HD folgt während des gesamten Ausschnitts mit seinem Blick genau GMs Gesten und Blicken. Dadurch kann er die Referenz von GMs elliptischen Zahlenangaben für sich klären. Allerdings scheint er Probleme bei der korrekten Erfassung der relevanten Zahlenwerte zu haben, wie sich in der Korrektursequenz in 16-25 zeigt: Zunächst formuliert HD 170 als Zielwert (19), was von GM durch 179 korrigiert wird (21); HD bestätigt mit der Nennung des Werts 79 (23), was erneut von GM durch 179 mit unterstützender Zeigegeste zum Plan korrigiert wird (24), HD wiederholt 79 (25). GM zeigt hier, dass er HDs Fehlverständnisse rezipiert, indem er sie korrigiert, er insistiert aber nicht auf einer letztlichen Klärung.

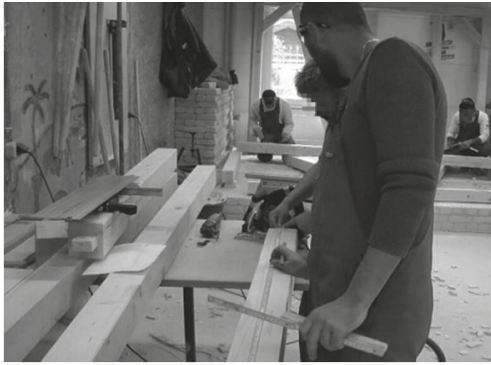

Bild 13 : „hundertNEUNzig“

(16)

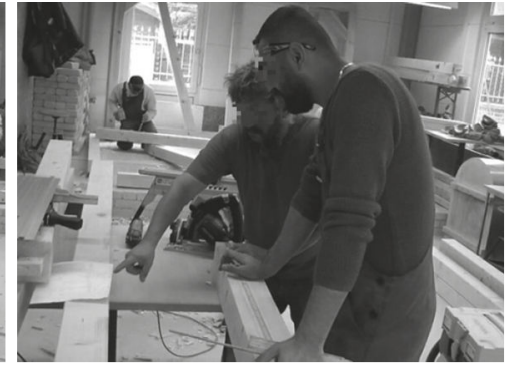

Bild 14: „wir BRAUchen hundertneunundsiebzig“ $(21)$

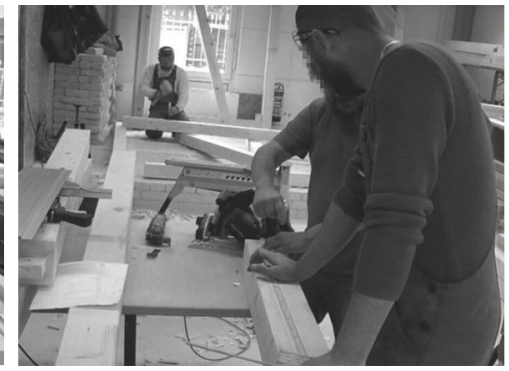

Bild 15: „das muss, ZWISCHen den beiden zApfen sein;" (26-27)

In 29 bis 41 erörtert GM, welche Längen die Zapfen an den beiden Seiten des Balkens haben sollen. Er nennt mögliche Werte für beide Seiten $(5,5,5,8,6)$ und summiert sie. GM prüft weder, ob HD die Zahlenwerte, noch ob er ihre Referenz (d.h. die Seiten des Balkens) und ihre Handlungsimplikativität (wo wie viel abzuschneiden ist) korrekt verstanden hat. Die Nennungen der möglichen alternativen Längen könnten HD verwirren; es wird weder deutlich, ob HD ihren Status verstanden hat, noch prüft GM sein Verständnis.

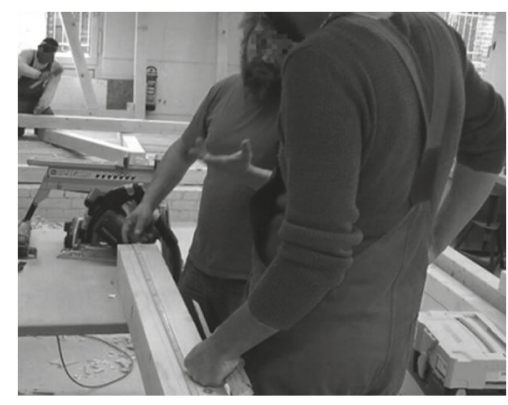

Bild 16: „hm?"“(54)

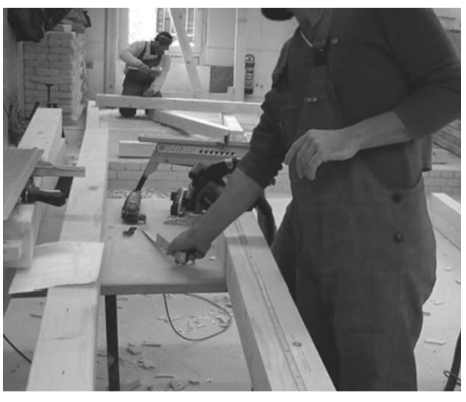

Bild 17: „oder fünf komma SIEben bei beiden. * (58)

Anschließend thematisiert GM implizit das Verhältnis zwischen Soll und Länge des Balkens (42-49). Der Themenwechsel und der damit verbundene Wechsel der Handlung (Überprüfung der Berechnung anhand der sichtbaren Abmessungen) werden sprachlich nicht verdeutlicht. Mit „also“ schließt GM in 51-52 einen Handlungsvorschlag an, der einer Instruktion an HD gleichkommt. Eingeleitet durch ein Achselzucken und mit ausgebreiteter linker Hand (54, Bild 16) zeigt GM an, dass es auch andere Möglichkeiten gäbe (vgl. Kendon 2004, S. 264-281) - eine davon formuliert er in 58. Währenddessen klappt 
HD seinen Zollstock ein (Bild 17) und verdeutlicht so, dass er die Sequenz für abgeschlossen hält.

Zusammenfassend können wir feststellen, dass der Ausbilder in diesem Ausschnitt fast ausschließlich Zahlenwerte thematisiert, die in Bezug auf vorverstandene Handlungsaufgaben und Zielvorgaben für ihre Durchführung verstanden werden müssen. Diese bleiben aber weitestgehend implizit. Ob HD diese Handlungsstrukturen kennt bzw. anhand von GMs Zahlennennungen (zusammen mit seinen referenziellen Gesten) rekonstruieren kann, bleibt unklar und wird von GM auch nicht zu prüfen versucht. Die teilweise fehlerhaften Zahlennennungen HDs lassen aber vermuten, dass sowohl fachliche als auch sprachliche Verständnisdefizite bestehen. Die Komplexität der sehr indexikalisch dargestellten Instruktionen wird weiter dadurch gesteigert, dass der Ausbilder häufig Alternativmöglichkeiten anspricht, die jedoch für einen fachlich nicht Vorgebildeten verwirrend sind. Es ist weder klar, wovon die Alternativen abhängen noch ob es Ermessensspielräume gibt und wie groß diese sind. Stattdessen mindern die Alternativformulierungen die Transparenz der Instruktion.

\subsubsection{Zusammenfassung der Interaktionsanalysen}

Die Analysen zu Interaktionen in den Werkstätten Holz und Metall der „PerF Plus“-Maßnahme verdeutlichen zwei Aspekte:

a) Die fehlenden Deutschkenntnisse auf Seiten der Flüchtlinge sind von grundlegender Bedeutung und beeinflussen jede Interaktion bis ins Detail.

b) Die Konzeption der Maßnahme geht aber von Praktikanten aus, die zumindest über ein Bl-Deutschniveau verfügen, d.h. erst nach der erfolgreichen Teilnahme an einem Integrationskurs so einer Maßnahme zugewiesen werden. An der Beteiligungsweise der beiden Ausbilder kann man diese Erwartungshaltung ablesen. Sie interagieren zumeist, als ob sie voll deutschkompetente Praktikanten vor sich hätten. Wenn sie aber adressatenspezifisch zu agieren versuchen, scheitern sie oft, da sie keine angemessenen kommunikativen Ressourcen einsetzen.

Folgende Aspekte charakterisieren Instruktionsinteraktionen in der von uns begleiteten „PerF Plus“-Maßnahme:

- Die Formulierung der Instruktionen weist oft einen mangelnden Adressatenzuschnitt (recipient design) auf: Instruktionen sind häufig syntaktisch und turn-strukturell zu komplex, vom Vokabular her zu anspruchsvoll und insbesondere zu fachsprachlich formuliert (\#2); sie sind zu implizit und indexikalisch, es fehlt eine grundlegende Darstellung der Aufgabenstrukturen und der Gelingenskriterien (\#4). Während insbesondere Zeigegesten erfolgreich zur Herstellung gemeinsamer Aufmerksamkeit und geteilter Referenz eingesetzt werden (alle analysierten Beispiele), sind illustrative, ikonische Gesten nicht immer erfolgreich (\#1, \#2), insbesondere, wenn sie diskrepant zum Verbalen oder als Vermittlungsinstrument semiotisch unterspezifiziert sind. Eine klare Turnstrukturierung mit einfacher Syntax, kongruenten illustrativen Gesten und klarer Handlungskategorisierung ist dagegen verstehensförderlich (\#1, \#2). Hilfreich sind ebenso Selbstreformulierungen und Erklärungen, die sensibel in Hinblick auf implizite Anzeichen von Verstehensproblemen seitens des Praktikanten (wie ausbleibende Rückmelder, Stirnrunzeln, Abbruch des Blickkontakts, falscher Blickfokus 
etc.) sind und versuchen, die Instruktion besser an die wahrscheinlichen fachlichen und sprachlichen Kenntnisse des Praktikanten anzupassen (\#2).

- Die Ausbilder prüfen kaum einmal systematisch das Verstehen der Praktikanten. Offenbar fehlt ihnen weitgehend eine zutreffende Einschätzung des Deutschniveaus der Praktikanten. Verstehensprobleme werden in den Instruktionssituationen selbst oft nur zufällig, durch offensichtlich unpassende Reaktionen der Flüchtlinge sichtbar (\#1, \#4). Da die Ausbilder weder deren Vorkenntnisse zu eruieren versuchen, noch während des Vermittlungsdiskurses didaktische Fragen stellen, zu lösende Aufgaben stellen oder Teile der Aufgabe von den Adressaten ausführen lassen, bleibt größtenteils im Dunkeln, was verstanden wurde. Dass Verstehen und damit auch Lernen nicht erfolgreich waren, stellt sich so zumeist erst viel später heraus, was dann dazu führt, dass Instruktionen umfassend wiederholt werden müssen oder gar die Vermittlung im Ganzen scheitert.

- Die Ausbilder nutzen die Ressource der Mehrsprachigkeit nicht adäquat. Switches ins Englische wie in \#1 und \#2 sind häufig nicht verstehensförderlich und tendenziell stigmatisierend. Dass Deutschkenntnisse unter den Praktikanten unterschiedlich verteilt sind und dass entsprechend einige systematisch als Dolmetscher für andere genutzt werden können, wird erstaunlicherweise von den Ausbildern nicht wahrgenommen, obwohl Dolmetschungen permanent stattfinden. Die Interaktion wird weder für Dolmetschbelange optimiert (in Bezug auf Adressierung und Turntaking) noch werden Dolmetschungen explizit ermutigt oder abgewartet.

- Bekundungen von Nichtverstehen oder Verstehensunsicherheiten und Rückfragen zur Verständnissicherung fehlen aber auch auf Seiten der Geflüchteten. In der Literatur zur Kommunikation von Muttersprachlern mit Nicht-Muttersprachlern ist es ein häufig genannter Aspekt, dass Letztere Verständnisschwierigkeiten oft gar nicht signalisieren, „entweder aus Angst, ,sein Gesicht verlieren“ zu können, oder als Strategie des Abwartens, die darauf hinausläuft, den Sinn einer Äußerung allmählich aus dem Kontext weiterer Äußerungen zu erschließen" (Bechtel 2003, S. 38f.). Ob die Praktikanten in unseren Aufnahmen aufgrund solcher „Let it pass“-Strategien (Firth 1996) ${ }^{20}$ oder auch aufgrund eines divergierenden kulturellen Verständnisses der angemessenen Beteiligungsweise eines Praktikanten im Lehr-Lern-Dialog auf Rückfragen verzichten, kann nicht mit Sicherheit geklärt werden. ${ }^{21}$

20 Firth (1996) hält anhand seiner Analysen von „Englisch bei Lingua franca-Sprechern“ fest, dass diese Strategie nur dann stattfindet, wenn die Aussage trotzdem verständlich ist oder wenn ein mangelndes Verständnis nicht zu fatalen Folgen in der Interaktion führt.

21 Schweigen im Gespräch kann abhängig von der jeweiligen Kultur Unterschiedliches bedeuten. Günthner (1993, S. 52) stellt bei ihrer Analyse von deutsch-chinesischen Interaktionen fest, dass im chinesischen Kontext „das Ausbleiben einer erwarteten Antwort, d.h. die Produktion von Schweigen, ein Kontextualisierungshinweis dafür ist, dass das Thema nicht erwünscht ist (Tabu) “. Die deutschen Gesprächspartner missverstehen das Schweigen aber regelmäßig als das Ausbleiben einer konditionell relevanten Antwort. 
Die zweite von uns begleitete Maßnahme „GASTRO“ fand von März bis August 2017 statt. 22 Wir suchten für unsere zweite Begleitstudie eine Maßnahme, die maximal mit der ersten kontrastiert. „GASTRO“ fand im Gegensatz zu „PerF Plus“ in einem Ballungsgebiet statt, was die Chancen erhöhte, dass mehr Teilnehmende vor Kursbeginn einen Integrationskurs besucht bzw. abgeschlossen haben. Außerdem haben die Initiatoren von "GASTRO" von Anfang an über die Arbeitsagenturen und Jobcenter gezielt Menschen angesprochen, die im Bereich der Hotellerie und Gastronomie arbeiten wollten, wodurch eine berufliche Passgenauigkeit eher gegeben war. Schließlich waren im Gegensatz zu den Ausbildern bei „PerF Plus“ die beiden Hauptverantwortlichen, also die sprachliche Lehrkraft (UH) und der gastronomische Ausbilder (FR), interkulturell ausgebildete und im Umgang mit Sprachlernern erfahrene Menschen.

„GASTRO“ geht auf die Initiative des Gastronomen FR zurück, der im Bereich Gastronomie und Hotellerie eine Qualifizierungsmaßnahme für Flüchtlinge anbieten wollte. Im Februar 2017 wurde die Auftaktveranstaltung der Maßnahme organisiert, zu der die interessierten Flüchtlinge über die Jobcenter und Arbeitsagenturen eingeladen wurden. Etwa 50 Teilnehmer besuchten die Auftaktveranstaltung. Davon wurden 24 für die beiden Assessmentwochen mit je zwölf Teilnehmern ausgesucht. Zwölf Teilnehmende wurden schließlich in die Maßnahme aufgenommen (fünf aus Gambia, vier aus Syrien und je einer aus Pakistan, Afghanistan und Eritrea). Kriterien waren, ob die Teilnehmer bereits in den Herkunftsländern in der Hotellerie und/oder Gastronomie gearbeitet hatten, sowie die Motivation der Teilnehmer, die sie in den Assessmentwochen gezeigt hatten. Ihre Deutschkenntnisse unterschieden sich stark voneinander. Während drei Teilnehmer bereits erfolgreich Integrationskurse absolviert hatten und Deutsch auf B1-C1-Niveau beherrschten, hatten neun Flüchtlinge keinen Sprachkurs besucht.

Nach den zwei Assessmentwochen bestand die Maßnahme aus einer achtwöchigen Praxisphase, welche mit Deutschunterricht begleitet wurde. Die Praxisphase und der Sprachunterricht wurden größtenteils in den Räumlichkeiten (Küche und Klassenzimmer) einer Berufsschule durchgeführt. Anschließend wurde allen Teilnehmern ein vierwöchiges Praktikum im Rhein-Neckar-Kreis vermittelt. Im Idealfall sollten sie nach Abschluss der Qualifizierungsmaßnahme dazu in der Lage sein, eine Arbeits- oder Ausbildungsstelle in einem Gastronomiebetrieb anzutreten.

\subsubsection{Interaktionsanalysen}

Unsere Analysen zu „PerF Plus“ in 4.1 haben deutlich gemacht, dass wegen fehlender Deutschkenntnisse ein Großteil der Teilnehmenden von den beruflichen Bildungsmaßnahmen nicht oder nur unzureichend profitiert. Nahezu jede Interaktion ist in gravierendem Maße durch sprachkompetenzbedingte Verstehens- und Verständigungsprobleme gekennzeichnet. Bei „PerF Plus“ waren die Ausbilder auf diese Situationen nicht vorbereitet, weshalb für alle Beteiligten sehr viele Interaktionen unbefriedigend verliefen. Wie wir im Folgenden sehen werden, gestalteten sich die Ausbildungsinteraktionen in „GASTRO“ oftmals produktiver. Der Ausbilder FR war sich nicht nur von Anfang an bewusst, dass ein

22 Unsere Videoaufnahmen von der Maßnahme umfassen knapp 12 Stunden. Wir konnten die Aufnahmezeitpunkte selbst wählen. Es wurde jeweils eine Woche am Anfang, in der Mitte und am Ende des Kurses aufgenommen. 
Teil der Teilnehmer praktisch keine Deutschkenntnisse besaß. Er hat es sich davon ausgehend zur Aufgabe gemacht, mit Hilfe verschiedener kommunikativer Strategien die fachliche Schulung systematisch mit der Sprachvermittlung zu verbinden. Anhand der folgenden Interaktionsanalysen zeigen wir, welche Praktiken er einsetzt und wie die Praktikanten auf seine Initiativen eingehen.

Mit \#5 analysieren wir zunächst eine Interaktion, die, wie bei „PerF Plus“, die grundlegende Problematik der fehlenden Deutschkenntnisse bei vielen Teilnehmern verdeutlicht. Der Ausbilder FR (Bild 18, links) erklärt zwei Praktikanten (RE (rechts) und NG (in der Mitte auf Bild 18)), wie sie einen Nachtisch vorbereiten sollen. Die Flüchtlinge sollen kleine Glasbecher mit 50 Gramm speziell zubereitetem Mangosaft etwa zu einem Drittel füllen. Dabei sollen sie darauf achten, dass die Ränder der Gläser sauber bleiben. Die aufgefüllten Gläser sollen dann wieder in Kartons gelegt werden, sodass der Saft abkühlen und hart werden kann. Am nächsten Tag soll dann eine von anderen Praktikanten vorbereitete Backpflaumencreme auf den Mangosaft kommen.

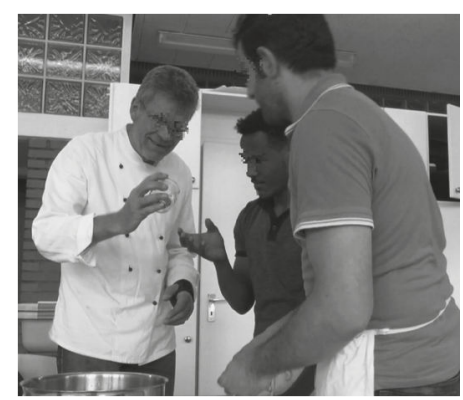

Bild 18: „GUCK mal“ (30)

\#5 Transkript „Sauber“ („GASTRO“, 3. Phase 1. Tag, 22.5.17, 00023, 0:09-2:19)

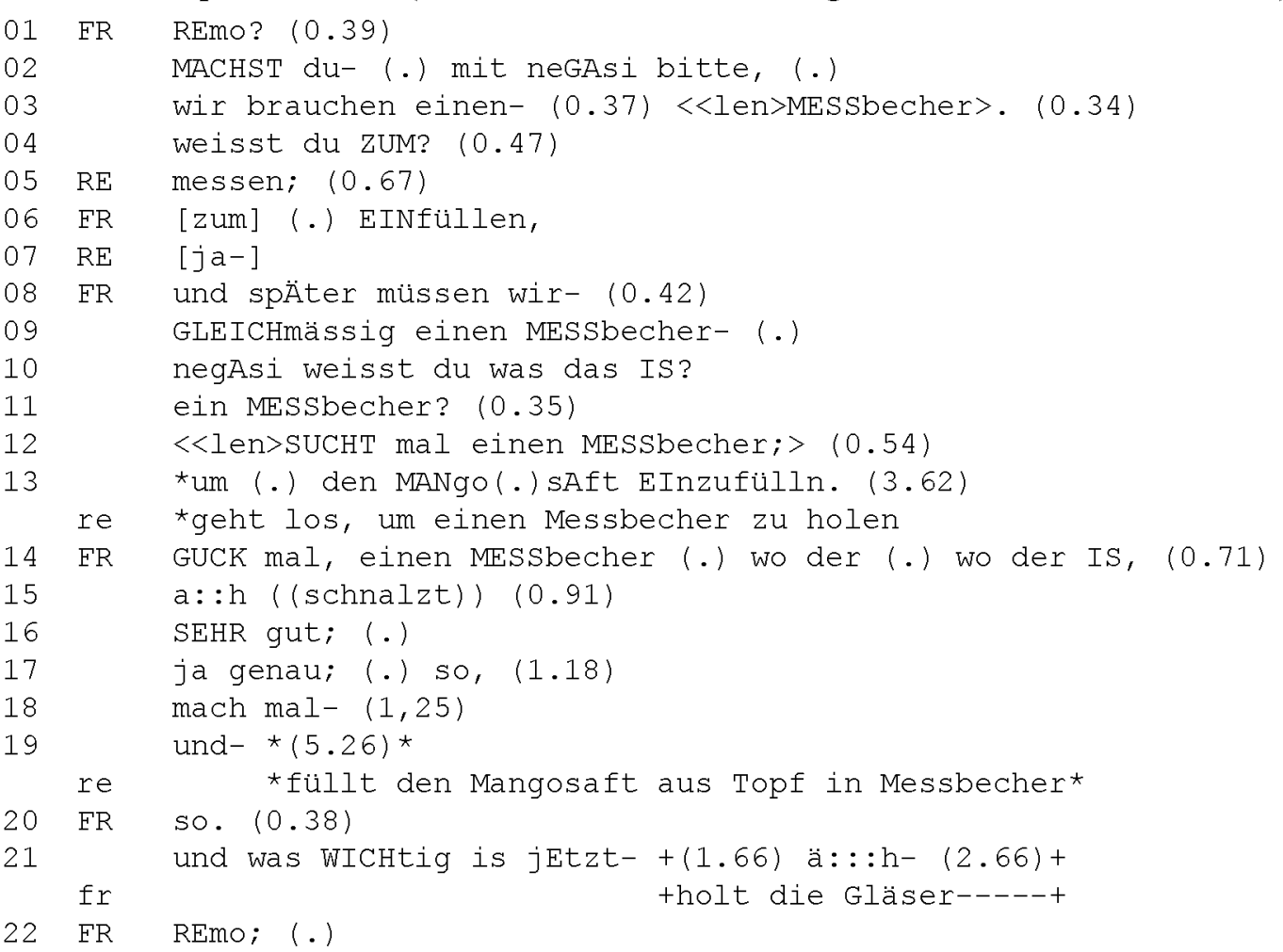


macht ihr zuSAMmen,

und was wICHtig is, (0.36)

$+\langle<a l 1\rangle$ warte wart wart; $>(0.23)+$

+zu NG, der sich ein Glas nehmen will+

< f>SAUber; > (.)

SAUber; +ja? $(0.74)+$ +nimmt ein Glas, stellt es gleich wieder ab+

$\langle<p+$ was is- $(0.39)$ thimmt ein zweites Glas hoch, betrachtet es und bewegt es Richtung

NG--------------------

----------------+ zeigt NG Kugel im Glas und lächelt---->

$<<l a ̈ c h e l n d>+G U C K$ \#mal, $>(0.55)+$ Sbewegt Hand Richtung Glas

---------++ bewegt Glas von NG zu sich + \#Bild 18

toKAY. (1.39)

+schütet Kugel aus dem Glas

((unverständlich)) (sauber) ((unverständlich))

so; talso NICHT- $(0.38)+$ thält Glas in linker Hand, zeigt auf dessen Rand + ich möchte nur- schAU mal- HIER; (.)

äh: :m + (4.18)+ +füllt Mangosaft mit Messbecher ins Glast fertig ja? (0.55)

FÜNEzig gramm; ich hol mal die WAAge, also (.) aber +bitte \#AUFpassen; +zeigt zeigefinger auf und ab bewegend auf Rand des Glases-------------------------> \#Bild 19

nicht- (.) ja, $(0.34)+$

es muss <<dim>sAuber bleibn. $>(0.64)$

$<<$ f das wird dann HART>, (.) das wird hArt in EIner stunde, wenn es KALT is,

( (Auslassung von 18 Sec.; Ausbilder beschreibt Creme, die auf den Mangosaft kommt, und gibt NG die Anweisung, nach dem Einfüllen des Safts alle Mangobecher in die Kartons zu füllen))

so. $(0.2)$

hol mal die WAAge, (.)

+die Gute wAAge von- (0.31)

fr tfasst NG an den schultern und schickt ihn Richtung waage

$<<$ len $>$ die WAAge. $>+$

ja? $\star(2.66)$

*nimmt Behälter mit Gläsern und geht nach hinten-----> bleIb- (.) ma bleIb doch DA, (1.01)*

SAUber machen? (0.29)

nein \#nein nein nein gut. \#Bild 20

nein?

nein nein. (1.56) 


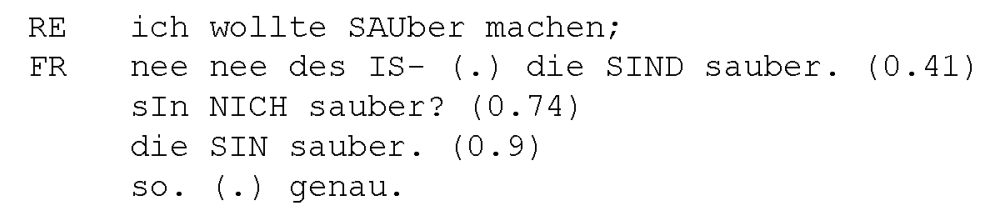

Zu Beginn der Interaktion spricht der Ausbilder FR den Praktikanten RE an und bittet ihn, den folgenden Arbeitsauftrag gemeinsam mit NG zu erledigen. Als Erstes richtet er an RE die Aufforderung, einen Messbecher zu besorgen: „REmo? (0.39) MACHST du (.) mit neGAsi bitte, (.) wir brauchen einen- $(0.37)<<1$ en $>$ MESSbecher $>$. (0.34) weisst du ZUM? (0.47) [...] (0.67) zum (.) EINfüllen" (01-06). Das zentrale Wort Messbecher wird vom Ausbilder FR in 03 partnersensitiv deutlich markiert: Es ist durch Pausen vom Turnkontext abgesetzt und wird durch langsameres Sprechtempo und Fokusakzent hervorgehoben. Der Praktikant RE bestätigt in 07 verstanden zu haben. Im Gegensatz zu ihm wohnt der zweite Praktikant NG relativ passiv und ohne Rückmeldesignale dem Geschehen bei. Vermutlich deswegen konfrontiert ihn der Ausbilder mit der Nachfrage: ,negAsi weisst du was das IS? ein MESSbecher?" (10-11) und fordert im Anschluss beide Flüchtlinge auf, einen Messbecher zu suchen (12-13). FR hebt die Handlungsaufforderung durch Pausen, langsameres Sprechtempo und Fokusakzente hervor: „(0.35) $<<$ len $>$ SUCHT mal einen MESSbecher; $>$ $(0.54)^{\text {“ }}$ (11-12). RE geht sofort los, um einen Messbecher zu holen. NG macht dagegen keine Anstalten, der Aufforderung nachzukommen, weshalb ihm gegenüber FR den Arbeitsauftrag ein drittes Mal wiederholt: „GUCK mal, einen MESSbecher (.) wo der (.) wo der IS,“ (14). Die Körpersprache NGs lässt unzweifelhaft erkennen, dass er entweder FRs Aufforderung etwas zu suchen oder den Gegenstand, wonach er suchen soll, nicht versteht: Er bleibt regungslos vor FR stehen. Inzwischen kommt RE mit einem Messbecher zurück an den Arbeitsplatz. Der Ausbilder FR quittiert die erfolgreiche Durchführung der Arbeitsanweisung mit explizitem Lob: „a::h ((schnalzt)) (0.91) SEHR gut; (.) ja genau; (.) so,“ $(15-16)$

Das explizite Loben der Praktikanten ist ein vom Ausbilder FR sehr häufig praktiziertes Rückmeldeverhalten. Solche positiven Feedbacks zeigen, dass sprachliche Defizite als normaler Ausgangspunkt angenommen und erfolgreiches Deutschlernen bzw. Beherrschung des Deutschen honoriert werden. Es ist anzunehmen, dass dies nicht nur das Selbstbewusstsein der Sprachlerner, sondern auch ihre Motivation und Bereitschaft, sich sprachliche und ausbildungsrelevante Inhalte anzueignen, stärkt. Bei „PerF Plus“ zeigte ein Ausbilder dieses partnersensitive Verhalten gar nicht, der zweite nur sporadisch.

RE füllt anschließend den Mangosaft aus dem Topf in den Messbecher. FR hebt gleich zu Beginn die Wichtigkeit seiner nächsten Anweisung hervor: „und was WICHtig is jEtzt(1.66) ä:...h- (2.66) REmo; (.) macht ihr zuSAMmen, und was WICHtig is, $(0.36)<<$ all $>$ warte wart wart; $>(0.23)<<\mathrm{f}>$ SAUber; $>$ (.) SAUber; ja? (0.74) was is- $(0.39)[\ldots]<<$

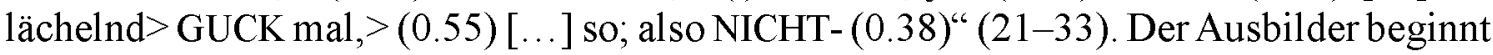
zunächst mit einer Relevanzhochstufung (,was WICHtig is jEtzt"), bricht diese Formulierung aber $a b$ und erinnert nach einer längeren Redepause, in der er die Gläser nach vorne auf dem Arbeitstisch holt, RE daran, dass er mit NG zusammenarbeiten soll („REmo; (.) macht ihr zuSAMmen"). Im Anschluss nimmt er die Relevanzhochstufung wieder auf (,was WICHtig is“), bricht vor dem fokussierten Skopus erneut ab und fordert NG auf, der bereits ein Glas in die Hand nehmen wollte, ihm zunächst zuzuhören (,warte, wart wart"). Erst danach kommt er zur zentralen Instruktion: „<<f> SAUber; $>$ (.) SAUber; ja?“ (26-27). 
Die gesamte Anweisung ist durch zahlreiche Abbrüche, Einschübe und Ellipsen gekennzeichnet. In 26-27 bleibt unklar, worauf sich „SAUber“ bezieht. Diese Uneindeutigkeit wird durch einen Vorfall verstärkt, der sich unmittelbar anschließt. Gegen Ende seiner Äußerung in 25 nimmt FR ein Glas in die Hand, das er aber gleich wieder ablegt. Stattdessen nimmt er ein anderes Glas hoch, in dem er ein Plastikkügelchen entdeckt hat: „,GUCK mal, (0.55) so; also NICHT- (0.38)“ (30-33). Leicht lächelnd zeigt er dabei NG die Plastikkugel im Glas (Bild 18) und schüttet sie dann zur Seite. Aufgrund dieser eingeschobenen Sequenz (28-31) könnte man denken, der Ausbilder habe mit „SAUber“ gemeint, dass die Praktikanten die Gläser vor dem Einfüllen sauber machen sollen. Der Ausbilder FR kontextualisiert aber die Sequenz mit dem Plastikkügelchen durch sein leichtes Lächeln als Nebensequenz (vgl. Jefferson 1972), die nicht zur Instruktion gehört. Der Verweis auf das Glas mit der Kugel durch den Ausbilder drückt also nicht aus, dass alle Gläser unrein sein könnten und sauber gemacht werden sollten. Das wird auch dadurch deutlich, dass der Ausbilder das betreffende Glas nicht weglegt, sondern zur Vorführung des nächsten Arbeitsschritts gleich im Anschluss verwendet. In 33 nimmt FR die Instruktion wieder auf mit dem Reformulierungsindikator „also NICHT“, der projiziert, was mit "SAUber“" in 26-27 gemeint war. Die Projektion wird aber verbal nicht eingelöst; durch das Zeigen auf den Rand des Glases lässt sich lediglich schließen, dass dies für die Erklärung dessen, was mit „SAUber“ gemeint war, von Bedeutung ist. FR füllt stattdessen das Glas mit Mangosaft und kommentiert dies verbal: ,ich möchte nur- schAU mal- HIER; (.) äh::m (4.18) fertig ja? (0.55)“ (34-36). Auch diese Äußerung ist abgebrochen, ihr fehlt wiederum das Rhema, das die neue Information zu beinhalten hätte. Erst jetzt, nachdem er den Arbeitsvorgang einmal manuell vorgeführt (aber nur sehr fragmentarisch erklärt) hat, kommt der Ausbilder zum Kern dessen, was er mit „SAUber“ meint (Bild 19): „FÜNFzig gramm; ich hol mal die WAAge, also (.) aber bitte AUFpassen; nicht- (.) ja, (0.34) es muss $<<\operatorname{dim}>$ SAUber bleibn.> (0.64)“ (37-41). Während seiner Äußerung zeigt er mit seinem Zeigefinger auf- und abbewegend auf den Rand des Glases. Mit "SAUber" meint der Ausbilder also, dass die Gläser beim Einfüllen innen an den Rändern sauber bleiben sollen.

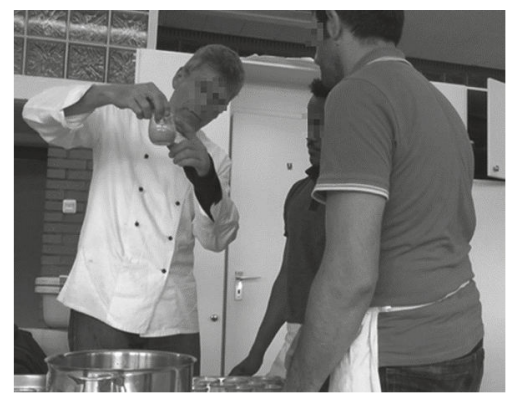

Bild 19: ,aber bitte AUFpassen“ (39)

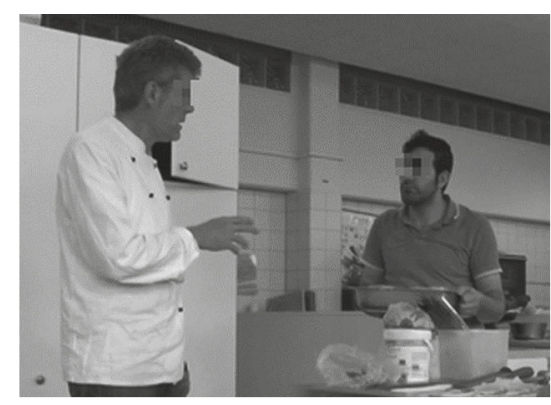

Bild 20: „nein nein nein nein gut.“" (57)

In den ausgelassenen folgenden 18 Sekunden beschreibt der Ausbilder den Praktikanten, wie später am Nachtisch weitergearbeitet wird. Nach der Beendigung der Instruktion geht der Praktikant RE mit dem Behälter mit den Nachtischgläsern nach hinten weg (54). Als ihn der Ausbilder auffordert, dazubleiben („,blEIb- (.) ma blEIb doch DA, “, 55), sagt RE, was er mit den Gläsern machen wollte: „SAUber machen? (0.29)“ (56). RE hat offensichtlich unter „sauber" verstanden, dass die Gläser vor der Befüllung mit der Mangomasse sauber zu machen sind. Der Ausbilder verneint dies (,nein nein nein nein gut", 57, Bild 20), woraufhin der Praktikant mit den Gläsern zurückkommt. Auf dem Weg zurück zum Tisch wieder- 
holt der Praktikant seine Absicht, die Gläser sauber machen zu wollen (60), worauf der Ausbilder widerspricht: „nee nee des IS- (.) die SIND sauber. (0.41)“ (61).

RE versteht die lexikalische Bedeutung von sauber, bezieht dies aber auf den Zustand der Gläser vor der Befüllung. Der Ausbilder sagte aber, dass die Gläser beim Einfüllen an den Rändern innen ,sauber bleiben“, d.h. keine Saftspuren zu sehen sein, sollen. Während die lexikalische Bedeutung und die grundsätzliche Referenz (die Gläser) keine Probleme bereiten, werden die genauere Referenz (welcher Teil der Innenfläche der Gläser ist gemeint?) und die Rolle des Ausdrucks im instruierten Handlungsprozess falsch verstanden. Das Missverstehen hat hier seine Ursache in der fragmentarischen Instruktion seitens des Ausbilders. Sie ist durch Abbrüche, Ellipsen, unmarkierte Referenzverschiebungen und Nebensequenzen gekennzeichnet. Gerade solche Instruktionen scheinen L2-Lernern große Probleme beim sprachlichen Verstehen zu bereiten.

In \#6 bereitet der Auszubildende LA (Bild 21, rechts) auf Anweisung des Ausbilders FR (Bild 21, links) eine Soße zu. Neben ihnen steht ein weiterer Teilnehmer (MU, rechts auf Bild 22), der ihre Interaktion zu Beginn beobachtet, während er ein eigenes Gericht zubereitet, aber nicht aktiv an der Interaktion teilnimmt.

\#6 Transkript „kochen oder köcheln“" (GASTRO“, 3.Phase 1. Tag, 22.5.17, 00031, $0: 37-1: 18)$

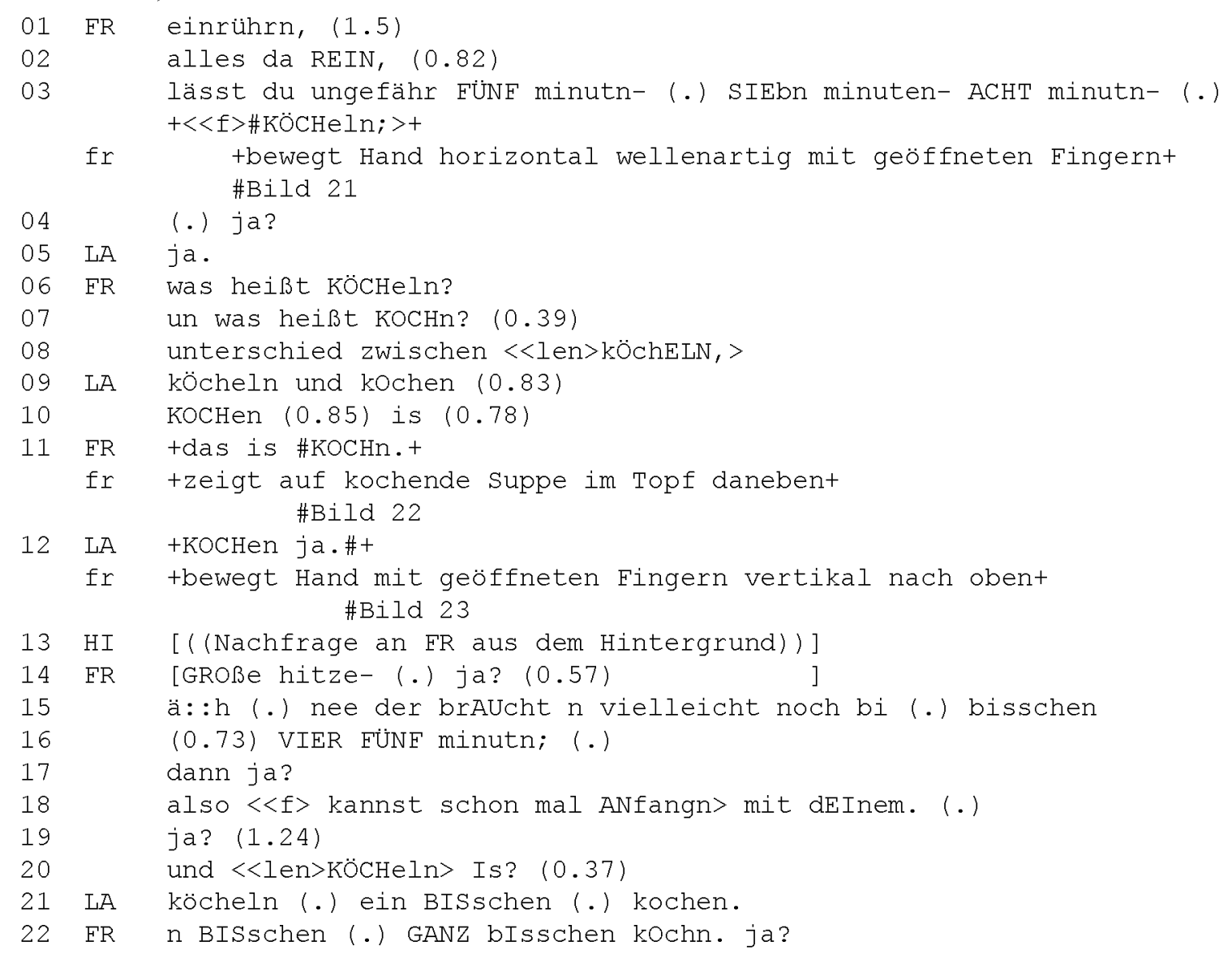

In dieser Sequenz steht der Praktikant LA am Herd und bereitet eine Gemüsesoße zu. Der Ausbilder FR unterstützt ihn mit verbalen Anweisungen: „einrührn, (1.5) alles da REIN, (0.82) lässt du ungefähr FÜNF minutn- (.) SIEbn minuten- ACHT minutn- (.) 
$+<<\mathrm{f}>$ \#KÖCHeln; $>$ (.) ja?“ (01-04, Bild 21). Das Verb köcheln ist für die Arbeit in der Küche ein sehr wichtiges Wort. Der Ausbilder verwendet auch hier die sprachlichen Gestaltungsmerkmale, die wir bereits in \#5 („MESSbecher", 03) bei der Einführung eines (vermutlich) neuen bzw. zentralen Worts gesehen haben: kurze Pausen vor und nach dem Ausdruck verbunden mit langsamerem Sprechtempo und Fokusakzent (,(.) $<<f>$ KÖCHeln; $>$ (.) ). Nach einer Rückversicherung, dass LA den Ausdruck rezipiert hat, die dieser bestätigt (04-05), nimmt der Ausbilder eine Wissensprüfung vor: „was heißt KÖCHeln? un was heißt KOCHn?" (06-07). Er zeigt damit, dass er damit rechnet, dass der Ausdruck von LA, der nur wenig Deutsch kann, nicht verstanden wird. Die Hervorhebung des Kontrasts von köcheln im Unterschied zu kochen und die Verstehensprüfung, anstelle der Fortführung der Instruktion, zeigt FRs Partnersensitivität und seine Strategie, die praktische Ausbildung mit der Sprachvermittlung zu verbinden. Eine vergleichbare Sequenz, in der verständnissichernde Rückfragen nach der Bedeutung eines Ausdrucks gestellt werden, war in der „Perf Plus“- Maßnahme bei keinem Ausbilder zu beobachten.

Mit köcheln und kochen werden zwei Verben miteinander kontrastiert, die semantisch und phonetisch nah beieinander liegen. Im Falle von kochen kann der Ausbilder mit großer Wahrscheinlichkeit davon ausgehen, dass die Flüchtlinge das Verb - zumal im Kontext der gastronomischen Maßnahme, in der es ständig benutzt wird - kennen. Weil die Verben ähnlich klingen, könnten die Flüchtlinge denken, köcheln und kochen seien gleichbedeutend. Durch die kontrastiven Fragen nach den beiden Verben macht der Ausbilder deutlich, dass es einen für die Arbeit relevanten semantischen Unterschied gibt.

Auf die Fragen reagiert LA zögerlich, zunächst mit einer Wiederholung der beiden Verben („kÖcheln und kOchen“, 09), dann mit dem Versuch, eine Antwort zu formulieren: „(0.83) KOCHen $(0.85)$ is $(0.78)^{\prime}$, 09-10). Sein Formulierungsproblem bzw. Wortsuchprozess tritt durch Disfluenzmerkmale, d.h. die vielen, relativ langen Pausen, deutlich zu Tage (vgl. Iványi 1998). FR beantwortet die Frage selbst mit einer Zeigegeste auf die kochende Suppe auf der Herdplatte und sagt: „das is KOCHn.“ (11, Bild 22). Da LA mit seiner zögerlichen Formulierungsarbeit deutlich machte, dass er mit der Beantwortung der Fragen Schwierigkeiten hat, ist die Antwort des Ausbilders an dieser Stelle gesichtsschonend. Er lässt den Praktikanten nicht länger zappeln und stellt ihn vor dem anderen Praktikanten MU nicht bloß.

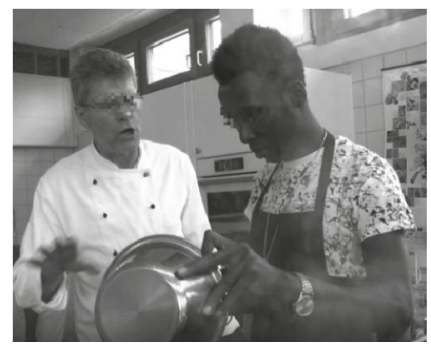

Bild 21: , $<<$ f $>$ KÖCHeln; $>$ (.) ja?" (03-04)

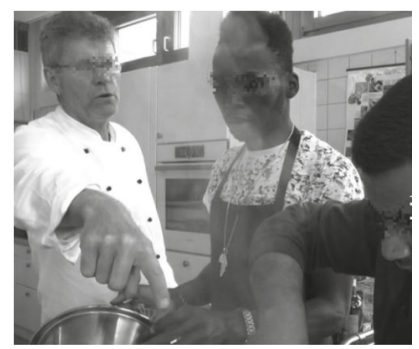

Bild 22: „das is KOCHn.“ (11)

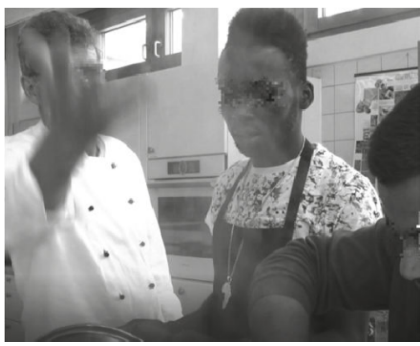

Bild 23: „KOCHen ja.“ (12)

Parallel zur folgenden Ratifizierung LAs („KOCHen ja.“, 12) verdeutlicht FR die Bedeutung von kochen durch eine illustrierende Geste, indem er seine Hand mit geöffneten Fingern vertikal nach oben bewegt. Kochen wird durch diese gestische Enaktierung (modeling, Streeck 2009, S. 119-150) mit starker Dampfentwicklung verknüpft (Bild 23). Bei der Arti- 
kulation des Verbs köcheln machte FR dagegen eine horizontal verlaufende, wellenartige Handbewegung mit geöffneten Fingern (03, Bild 21). Der Kontrast der Handbewegungen (vertikal vs. horizontal) verdeutlicht die unterschiedliche Intensität der beiden Kochaktivitäten. Im direkten Anschluss liefert FR auch eine verbale Paraphrase: „GROße hitze- (.) ja?“ (14).

Nach einem kurzen Nebengespräch mit einem anderen Teilnehmer, HI, über die Zubereitung dessen Gerichts (13-19) fragt FR LA: „und $<<$ len $>$ KÖCHeln $>$ Is?" (20). LAs Antwortversuch ist diesmal erfolgreicher: „köcheln (.) ein BISschen (.) kochen“(21). LA bietet eine Paraphrase für köcheln, die die von FR vorgegebenen Begriffe zueinander ins Verhältnis setzt. Die Sequenz abschließend wiederholt der Ausbilder LAs Antwort nur mit geringer Abänderung (durch die Gradpartikel ,ganz") und explizit ratifizierendem ,ja“ (,n BISschen (.) GANZ bIsschen kOchn. ja?“", 22).

Der Ausbilder FR nimmt während der gesamten Maßnahme jede sich bietende Gelegenheit wahr, um mit den Flüchtlingen die fachliche Wissensvermittlung mit Sprachvermittlung im Sinne eines ,content and language integrated learning“ (CLIL, Marsh 1994) zu verbinden. ${ }^{23}$ Mit Hilfe von Paraphrasierungen und Kontrastierungen gelingt es ihm, Fachausdrücke aus der Gastronomie einzuführen und den Flüchtlingen verständlich zu erklären. ${ }^{24}$

Im folgenden Ausschnitt \#7 aus der gastronomischen Maßnahme geht es um eine Instruktion, wie sie der Ausbilder an jedem Arbeitstag zu Beginn der Rezeptbesprechungen durchführt. FR hat alle Praktikanten im Kochunterrichtsraum um eine Tischgruppe versammelt (Bild 24). Er evaluiert frühere Kochaktivitäten und gibt Instruktionen für die anstehenden. Im vorliegenden Fall hatte einer der Praktikanten am Tag zuvor die Aufgabe, Bohnen zu kochen. Es geht nun darum, wann man beim Kochen von Bohnen Salz hinzugeben sollte, damit sie schneller weich werden.

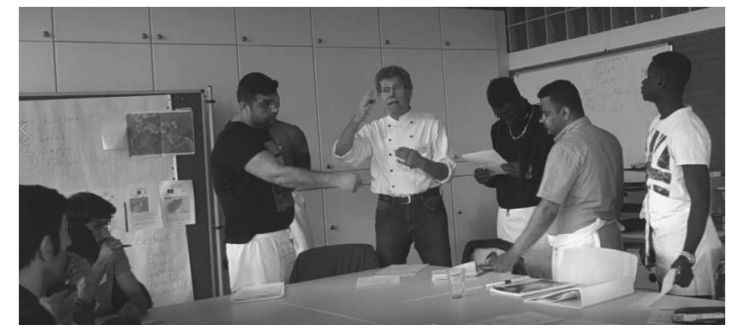

Bild 24: „das <<len> GE:genteil> von wEIch?“ $(07)$

\#7 Transkript „,̈̈bersetz mal für rozan“ („GASTRO“, 1. Phase 3. Tag, 29.3.17, 00008, $7: 14-8: 33)$

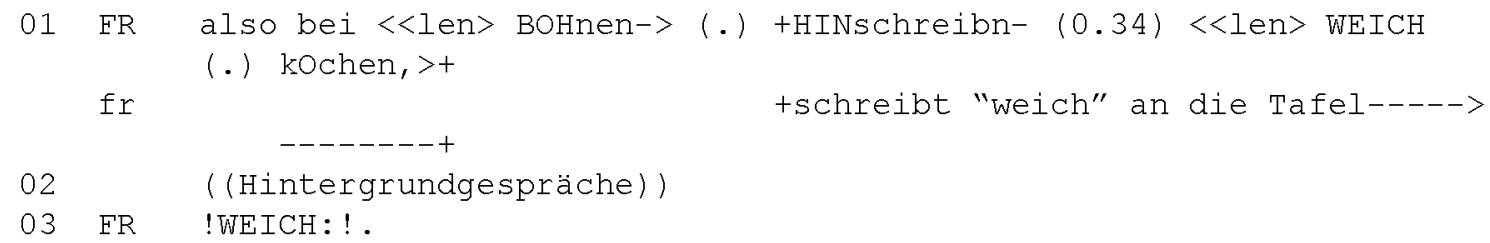

23 In einem anderen Fall stand der Ausbilder z.B. bei einem Flüchtling, der gerade spülte. Er nutzte diese situative Möglichkeit und fragte jedes Mal, wenn der Praktikant etwa einen Topf oder ein Sieb abwusch, nach dem Namen des Gegenstands und korrigierte anschließend, falls notwendig, seine Aussprache.

24 So fragt er etwa zu einem anderen Zeitpunkt den gleichen Praktikanten LA, ,wie bindest du eine Soße?“. Nachdem der Ausbilder erkennt, dass der Flüchtling diesen Fachausdruck nicht erkennt, reformuliert er seine Frage mit einer Paraphrase: „Wie machst du die Soße dick?" 


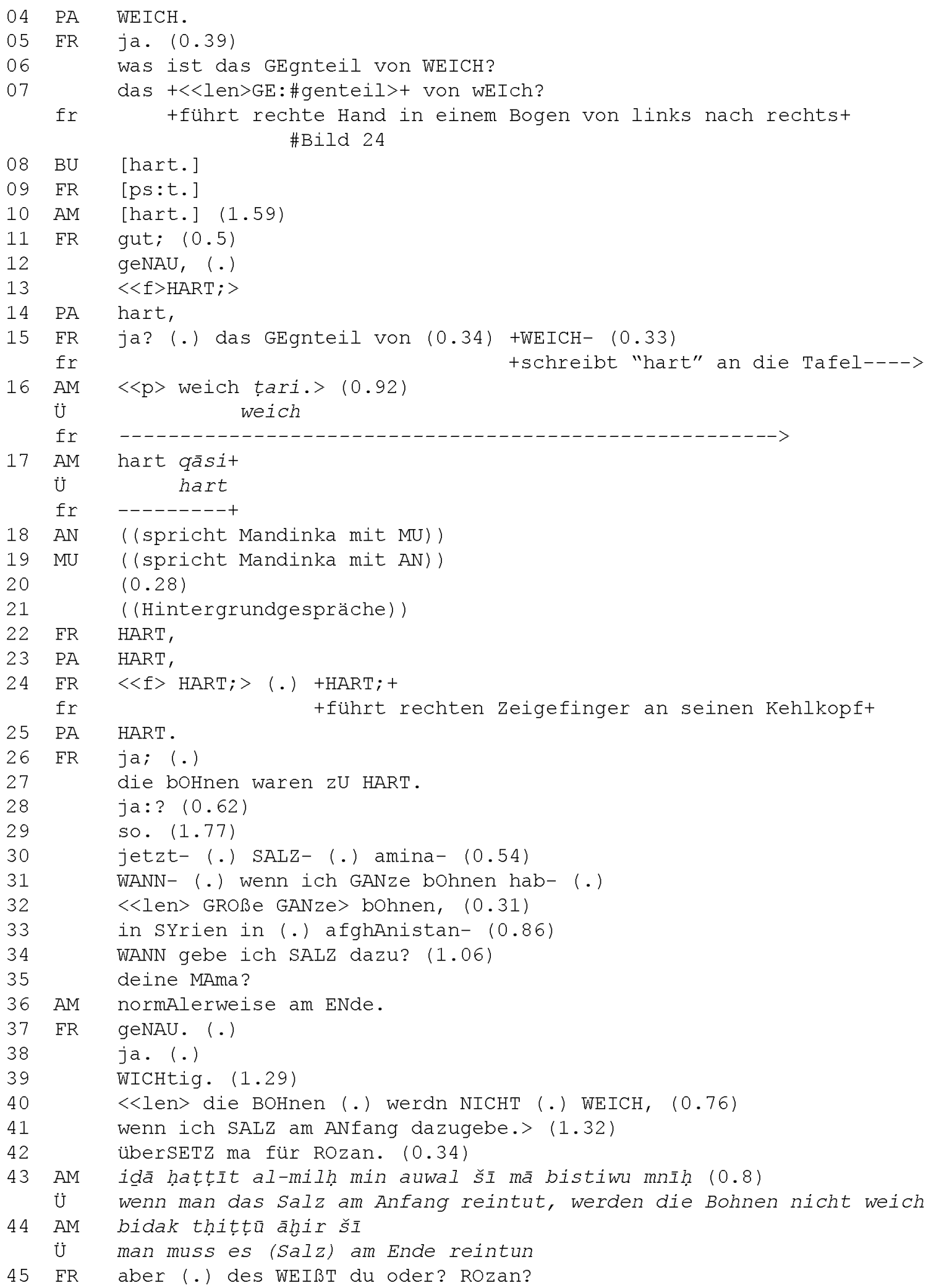

Am Tag zuvor hatte einer der Praktikanten die Aufgabe, weiße Bohnen zu kochen, die nun weiterverarbeitet werden sollten. Da die Bohnen aber nicht weich genug wurden, bespricht der Ausbilder mit den Praktikanten die Gründe dafür. Alle Teilnehmer haben das Rezept mit den Bohnen vor sich. Gleich zu Beginn fordert der Ausbilder die Praktikanten auf, auf 
ihre Rezepte „Bohnen weich kochen“ (01) zu schreiben. Dabei schreibt er „weich“ an die Tafel (auf Bild 24 rechts hinten). Auf diese Möglichkeit, Wörter und Ausdrücke an die Tafel zu schreiben, greift FR immer wieder zurück. ${ }^{25}$ Obwohl auch in den Werkstätten von „PerF Plus“" Flipcharts und Tafeln vorhanden waren, wurden sie dort nie von den Ausbildern als Lernhilfe genutzt. Bei „GASTRO“ ist auch diese Praktik Teil der Strategie des Ausbilders, die Vermittlung von Fachkenntnissen mit Sprachvermittlung zu kombinieren.

Der Ausbilder fragt anschließend die Flüchtlinge nach dem Gegenteil von weich (06). Er wiederholt seine Frage mit illustrierender Gestik: Während er in langsamerem Sprechtempo und mit Fokusakzent ,das $<<1$ en $>$ GE:genteil $>$ von wEIch?" fragt, bewegt er seine rechte Hand in einem hohen Bogen von links nach rechts (07) und verdeutlicht somit mit dieser diskursiven Geste (vgl. Müller 1998, S. 112), dass eine Gegensatzrelation gesucht ist. Wie oben ausgeführt, haben drei der Teilnehmer bereits erfolgreich einen Integrationskurs besucht und verfügen über Deutschkenntnisse auf mindestens B1-Niveau. Zwei von ihnen (AM und BU) antworten nun gleichzeitig auf die Frage des Ausbilders: „hart" (08 und 10). Der Ausbilder versucht sie mit „ps:t“ (09) an der Antwort zu hindern, damit die anderen, die noch nicht so gut Deutsch können, auch eine Gelegenheit haben, zu antworten. Dennoch lobt er sie für die richtige Antwort und wiederholt das gesuchte Wort wiederum abgesetzt und mit erhöhter Lautstärke: ,gut; (0.5) geNAU, (.) $<<\mathrm{f}>$ HART; >“ (11-13). Auch dieses Wort schreibt er dann im Anschluss an die Tafel (15). Währenddessen wendet sich AM, Flüchtling aus Syrien, der Deutsch auf B1-Niveau beherrscht, zu den anderen arabischsprachigen Flüchtlingen und dolmetscht ihnen unaufgefordert die beiden Adjektive ins Arabische: „weich tari (0.92) hart qāasi“ (16-17).

Als der Ausbilder FR das Adjektiv erneut ausspricht („HART,“ 22), nutzt PA (Flüchtling aus Gambia mit Deutschkenntnissen auf B1-Niveau), der hin und wieder Probleme mit der Aussprache hat, die Gelegenheit, die korrekte Aussprache des Wortes zu üben (23 und 25). FR unterstützt ihn dabei, indem er „HART“ noch zweimal vorspricht (24) und seinen Kehlkopf berührt, um auf die Vibration des Konsonanten /r/ hinzuweisen. ${ }^{26}$

Schließlich kommt der Ausbilder nach der langen sprachdidaktischen Einschubsequenz zur fachlichen Instruktion. Er wiederholt: ,ja; (.) die bOHnen waren zU HART." (26-27). Anschließend thematisiert er den Grund dafür in Form einer didaktischen Frage an AM, wann man beim Kochen von Bohnen das Salz dazu geben sollte: ,jetzt- (.) SALZ- (.) amina- (0.54) WANN- (.) wenn ich GANze bOhnen hab- (.) $<<$ len $>$ GROße GANze $>$ bOhnen, (0.31) in SYrien in (.) afghAnistan- (0.86) WANN gebe ich SALZ dazu? (1.06) deine MAma“ (30-35). Mit dem Bezug auf die kulturelle (,in SYrien in (.) afghAnistan“) und familiäre Herkunft (,deine MAma“) stellt FR hier AM die Frage, wann beim Kochen von Hülsenfrüchten das Salz dazugegeben wird. FR zeigt damit, dass er das von den Flüchtlingen biografisch erworbene Wissen würdigt und dass dieses zur Bewältigung der sich in der Ausbildung stellenden Aufgaben wertvoll ist. Der Ausbilder spricht bei Fragen, die spezifisches Fach- oder Sprachwissen benötigen, häufig AM an, da dieser sowohl Deutsch auf B1-Niveau beherrscht als auch in der Gastronomie erfahren ist. Das zeigt, wie genau der Ausbilder die Kompetenzen der Teilnehmer kennt und wie er diese partnersensitiv einsetzt.

25 Schon am Anfang des Kurses wurden bei „GASTRO“ an die Teilnehmer Blöcke und Stifte verteilt, damit sie wichtige Wörter, Ausdrücke etc. notieren können.

26 Vgl. Svennevig (2018, S. 2) zu Wiederholungen als manifeste Orientierung auf eine gemeinsame pädagogische Aktivität. 
Der Praktikant AM liefert die richtige Antwort auf FRs Frage („,normAlerweise am ENde.“, 36) und bekommt dafür eine positive Rückmeldung vom Ausbilder: „geNAU. (.) ja. (.)“ (37-38). Anschließend wiederholt FR die Instruktion in negierter Form, indem er prospektiv zunächst die Relevanz der kommenden Äußerung hochstuft: „WICHtig. (1.29) $<<$ len> die BOHnen (.) werdn NICHT (.) WEICH, (0.76) wenn ich SALZ am ANfang dazugebe. ${ }^{\text {" }}$ (39-41). FR zeigt eine sehr rekurrente Formulierungsweise bei Instruktionen, mit der er wichtige Aspekte hervorhebt: Er stuft explizit die Relevanz hoch (,wichtig“), verlangsamt das Sprechtempo, segmentiert seinen Beitrag in mehrere kleine Informationseinheiten mit Hilfe vieler Pausen und hebt mit Fokusakzenten die wichtigsten Wörter hervor („WICHtig, BOHnen, WEICH, SALZ, ANfang“; vgl. Svennevig 2015). Dadurch fördert er, dass L2-Lerner diese wichtigen Inhalte leichter verstehen. In der Folge benutzt der Ausbilder ein weiteres Verfahren, um wichtige Inhalte auch den Sprachlernern im Kurs zugänglich zu machen: Der Ausbilder fordert AM, der gut Deutsch kann, auf, für RZ, einen anderen syrischen Teilnehmer, der kaum Deutsch kann, seine letzte, inhaltlich wichtige Äußerung ins Arabische zu dolmetschen: „überSETZ ma für ROzan.“ (42). Der Ausbilder setzt die Deutschkompetenzen eines Teilnehmers (AM) als Ressource ein, damit Wissen an die weniger deutschkompetenten Mitglieder der Praktikantengruppe auch jenseits der durch seine eigenen Sprachkenntnisse gesetzten Grenzen vermittelt werden kann. Wie wir bereits vorher (16-17) sehen konnten, dolmetscht AM auch unaufgefordert für die anderen syrischen Teilnehmer. Ähnliches konnten wir auch schon bei einem der Teilnehmer der „PerF Plus“-Maßnahme beobachten (s.o. \#3). Im Unterschied dazu nutzt bei „GASTRO“ der Ausbilder die Übersetzungskompetenzen gezielt als Ressource, während es bei „PerF Plus" den Ausbildern offenbar unbekannt war, dass einzelne Teilnehmer Deutsch auf B1-Niveau beherrschten und sie diese als Dolmetscher einsetzen könnten.

Im Rahmen von „GASTRO“ verknüpft der Ausbilder die fachliche Vermittlung immer wieder systematisch mit der Deutschvermittlung. Dazu initiiert er schultypische InitiativeResponse-Evaluation-Sequences (I-R-E, Mehan 1979), in denen er Fragen nach dem sprachlichen Wissen der Kursteilnehmer stellt und dieses im dritten Zug des Sequenzmusters, der Evaluation, nochmals in autoritativer Form laut und deutlich wiederholt und (wie in \#7) manchmal auch an die Tafel schreibt. Das Sprachlernen wird somit immer wieder zu einem eigenständigen Vermittlungsfokus, der aber stets motiviert ist durch die praktischen Lern- und Handlungsaufgaben und auch an sie zurückgebunden wird. Dabei greift der Ausbilder immer wieder auf die Deutschkompetenzen von AM zurück, um wichtige Inhalte ins Arabische übersetzen zu lassen. Eine andere Strategie des Ausbilders ist, die eigene Mehrsprachigkeit als Ressource zu nutzen. Er hat lange Zeit in Lateinamerika und Asien gearbeitet, weshalb er über sehr gute Kompetenzen im Englischen und Spanischen verfügt. Codeswitches ins Spanische benutzt der Ausbilder mit einem Flüchtling aus Gambia, der, bevor er nach Deutschland kam, fünf Jahre in Spanien gelebt hatte. In Interaktionen mit allen anderen wechselt er sehr häufig ins Englische, wenn er wichtige Begriffe einführen oder erklären will. Sprachwechsel werden von FR stets funktional und partnersensitiv eingesetzt.

Im letzten Beispiel \#8 aus der „GASTRO“-Maßnahme werden die zu kochenden Rezepte für den betreffenden Tag verteilt und mit den Praktikanten durchgegangen. FR bespricht ein Rezept für vier Personen. Da aber die Gruppe einschließlich Ausbilder aus zwölf Personen besteht, müssen die Praktikanten das Rezept bei der Kalkulation mit drei multiplizieren. 


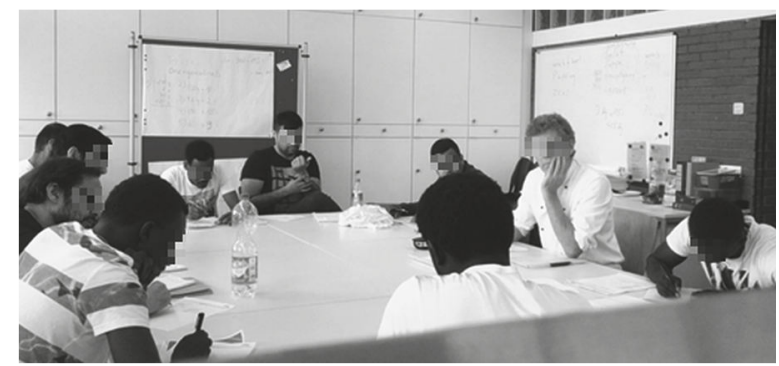

Bild 25: ,jetzt DREI mal das rezept- (1.39)“ (19)

\#8 Transkript „rezept mal drei““ („GASTRO“, 1. Phase 4. Tag, 00010, 10:23-11:14)

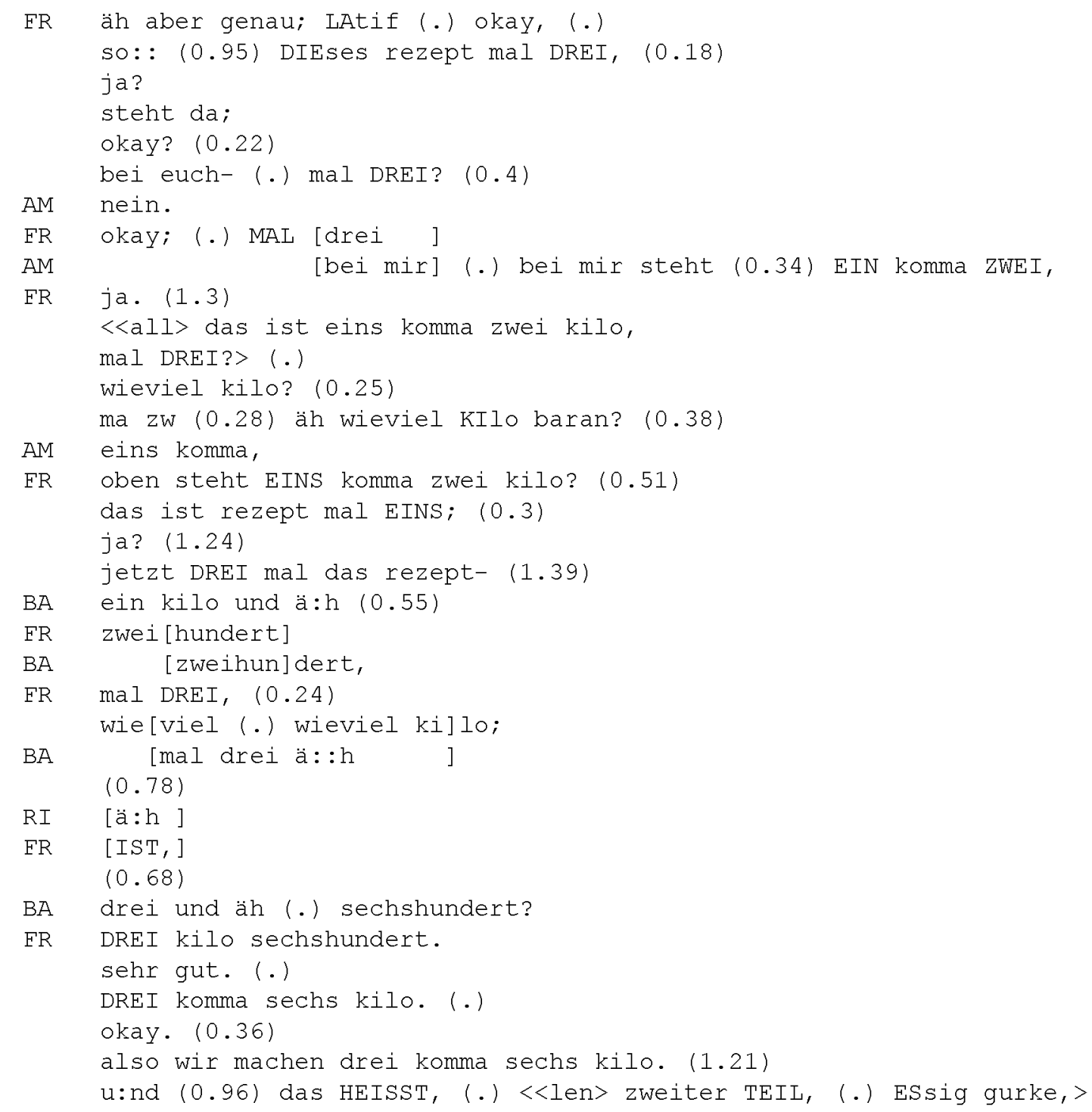

Da das Rezept für vier Personen ausgelegt ist (mit dem Gesamtgewicht von 1,2 kg), die Flüchtlinge aber für zwölf kochen sollen, müssen alle Mengenangaben mit drei multipliziert werden. Der Ausbilder hatte schon während der Assessmentwochen zu Beginn der Maßnahme bemerkt, dass die Teilnehmer sprachliche Probleme mit Zahlen, Mengenangaben und Rechenoperationen haben. Bei „PerF Plus“ haben die Ausbilder diesen Aspekt größtenteils nicht wahrgenommen bzw. in keinem Fall explizit thematisiert oder bearbei- 
tet, wie wir auch in \#4 sehen konnten. In \#8 widmet sich der „GASTRO“-Ausbilder dieser Problematik dagegen explizit.

Nachdem die Beteiligten geklärt haben, dass auf den Rezepten nicht, wie vom Ausbilder erwartet, festgehalten ist, dass die Angaben mit drei multipliziert werden müssen (01-09), stellt der Ausbilder die Rechenaufgabe ,rezept mal DREI“ (02) zunächst an alle, dann an den Teilnehmer BA: ,ja. (1.3) <<all> das ist eins komma zwei kilo, mal DREI?> (.) wieviel kilo? (0.25) ma zw (0.28) äh wieviel KIlo baran?" (10-14). Als keine Reaktion erfolgt, reformuliert er die Aufgabe, indem er sie in drei Schritte aufgliedert: „oben steht EINS komma zwei kilo? (0.51) das ist rezept mal EINS; (0.3) ja? (1.24) jetzt DREI mal das rezept- (1.39)“ (16-19). Die Reformulierung macht die Aufgabe transparenter, indem sie sie in Teilschritte zerlegt und gibt dem angesprochenen Flüchtling BA mehr Zeit, sich auf die Frage einzustellen. In dessen Formulierung zeigt sich in der Folge an Häsitationen und Pausen sehr deutlich, dass BA Probleme mit der Aufgabenstellung hat: „ein kilo und ä:h $(0.55)^{\prime \prime}(20)$. Der Ausbilder kommt zu Hilfe, ergänzt zunächst die Zahl (,zweihundert“, 21), und stellt dann die Rechenaufgabe nochmal: „mal DREI, (0.24) wieviel (.) wieviel kilo;" (23-24). Parallel zum Ausbilder wiederholt BA dessen Äußerungen ,zweihundert" (22) und „mal drei ä::h“ (25) und liefert dann, wieder begleitet von Häsitationssignalen (27) und Pausen (26 und 29), etwas verzögert die richtige Antwort: „drei und äh (.) sechshundert?" (30). BAs Antwort und seine Formulierungsarbeit zeigen, dass für ihn größere Zahlen und Rechenoperationen, wie generell für viele Flüchtlinge, ein sprachliches Gebiet sind, das sie noch einüben müssen. Die Fragesequenz beendet der Ausbilder, indem er die Antwort zunächst ergänzend wiederholt („DREI kilo sechshundert.“, 31) und den Flüchtling für seine richtige Antwort explizit lobt (,sehr gut.“, 32), dann aber nochmals in kanonischer Form (,DREI komma sechs kilo.“, 33) reformuliert.

Wiederum nutzt FR hier das Verfahren der I-R-E-Sequenz, um das Wissen der Flüchtlinge zu aktivieren, zu prüfen und zu korrigieren. Eine wichtige Rolle spielt dabei auch die jeweils abschließende explizite Ergebnissicherung, die das für alle verbindliche, zu vermittelnde Wissen festhält.

Bei „PerF Plus“ hatten wir bei der Besprechung des Transkripts \#4 gesehen, dass der Ausbilder Zahlen ohne Erklärung und Verstehensprüfung verwendete und Rechenoperationen selbst durchführte. Sein Vorgehen ließ sich dahingehend interpretieren, dass er die Probleme der Sprachlerner mit Zahlen und Rechenoperationen in der praktischen Ausbildung nicht adäquat wahrnimmt und bearbeitet. Gerade im handwerklichen Bereich stellt aber der Umgang mit Zahlen, Mengenangaben und Rechenoperationen eine wesentliche Kompetenz dar. Bei „GASTRO“ geht der Ausbilder angesichts der (teils antizipierbaren, teils manifesten) sprachlichen Probleme der Flüchtlinge auf diesem Gebiet diese Aufgabe dagegen proaktiv an. Wie man in \#8 sehen kann, macht er aus der Rezeptbesprechung einen mathematischen Sprachunterricht und nutzt die Gelegenheit, Zahlen und Rechenoperationen auf Deutsch einzuüben.

\subsubsection{Zusammenfassung der Interaktionsanalysen}

Fehlende Deutschkompetenzen der Flüchtlinge sind für die Maßnahme „GASTRO“ ebenso prägend wie für „PerF Plus“. Bei beiden Maßnahmen kam es häufig zu Interaktionen, in denen die Geflüchteten den Anweisungen und Erläuterungen sprachlich nicht folgen konnten. Der „GASTRO“-Ausbilder FR benutzt aber im Gegensatz zu den Ausbildern von „PerF Plus“ ein breites Repertoire sprachlich-kommunikativer Strategien, um mit Ver- 
ständigungsproblemen umzugehen. Er verwendet darüber hinaus spezielle Strategien, um (Fach-)Sprachvermittlung zu betreiben. Zusammenfassend zeichnet sich sein Repertoire durch folgende Eigenschaften aus:

- In der Regel schafft es der Ausbilder, seine Instruktionen mit passgenauem Adressatenzuschnitt zu formulieren: Wichtige Begriffe hebt er durch verlangsamtes Sprechtempo, Lautstärke, Fokusakzente und Pausen vor und nach der fokussierten Stelle hervor (\#5, \#6, \#7). Seine Formulierungsweise ist durch klare Turnstrukturierung mit einfacher Syntax und einer klaren Kategorisierung und Explikation der zu vollziehenden Handlungen charakterisiert (\#6, \#7, \#8). Verstehensunterstützend werden von ihm sequenziell eindeutige Zeigegesten und seine Turns illustrierende Gesten eingesetzt (\#5, \#6, \#7). Auf Verstehensprobleme der Praktikanten wie ausbleibende Handlungsdurchführung reagiert er mit Selbstreformulierungen und Nachfragen (\#5).

- FR betreibt neben Fachausbildung auch Sprachvermittlung (CLIL). Er nutzt nahezu jede Gelegenheit (z.B. beim Kochen, Spülen etc.), den Flüchtlingen relevantes (Fach-) Vokabular beizubringen oder, falls es schon eingeführt war, in I-R-E-Sequenzen nach- bzw. abzufragen. Sie dienen dem Ausbilder systematisch dazu, das Vorwissen und das Verstehen der Praktikanten zu prüfen. Da dabei häufig artikulatorische Schwierigkeiten zu beobachten sind, wiederholt er die didaktisch abgefragten, für den jeweiligen Arbeitszusammenhang zentralen Ausdrücke langsam und standardnah und animiert so die Teilnehmer zur Einübung der Aussprache (\#7). Dabei sind seine Fragen wie auch die Ergebnissicherungen der I-R-E-Sequenzen so gestaltet, dass nicht nur der jeweilige Antwortende, sondern die gesamte Gruppe adressiert wird. Oft im Anschluss an Ab- und Nachfrage-Sequenzen, wenn die Teilnehmer die Antwort nicht liefern, aber nicht selten auch proaktiv, greift der Ausbilder zur Verständnissicherung neben gestischen Mitteln vor allem auf Paraphrasierungen und die Zerlegung der Instruktion in einzelne Schritte und minimale Turnkonstruktionskomponenten zurück (\#6). Gerade in beruflichen Kontexten, in denen das Erlernen von Fachvokabular unumgänglich ist, ist die Umschreibung und Erklärung von fachsprachlichen Ausdrücken mit einfachen Alltagsbegriffen eine hilfreiche und effektive Methode (vgl. Svennevig et al. 2017). Die sprachdidaktischen Sequenzen nehmen ebenso wie die didaktischen Strategien der Fachvermittlung ihren Ausgang stets von grundlegenden praktischen Aufgaben, auf die sie immer wieder zurückbezogen werden. Das Sprachlernen bleibt daher nicht abstrakt, sondern wird an die fachlichen Ausbildungserfordernisse und die praktischen, unmittelbar im Anschluss umzusetzenden Handlungen der Praktikanten angebunden.

- Der Ausbilder FR hat gleich zu Beginn der Maßnahme genau eruiert, über welche Deutsch- und andere fremdsprachigen Kenntnisse die einzelnen Teilnehmer verfügen. So nutzt er adäquat die fortgeschrittenen Deutschkenntnisse einiger Teilnehmer, um auch durch systematische Dolmetschungen wichtige Inhalte des Kurses anderen Teilnehmern zukommen zu lassen (\#7). Ebenso nutzt er sehr effektiv die Ressource seiner eigenen Mehrsprachigkeit. Da der Ausbilder über gute Kenntnisse im Spanischen und Englischen verfügt, wechselt er adressatenspezifisch in diese Sprachen, um einzelnen Teilnehmern, die auch Kompetenzen in diesen Sprachen besitzen, verstehensförderliche Dolmetschungen anzubieten.

Zusammenfassend lässt sich sagen, dass das kommunikative Verhalten des Ausbilders ein sehr gutes Beispiel für eine erfolgreiche integrierte Fach- und Sprachausbildung ist. 


\section{Schluss und Ausblick}

Die auf nationaler Ebene formulierte „Willkommenskultur“ (siehe Einleitungsartikel in diesem Heft) muss sich derzeit auf lokaler Ebene in Maßnahmen zur beruflichen Eingliederung bewähren. Die hohe Anzahl von Flüchtlingen, die seit 2014 nach Deutschland gekommen sind, aber auch eine Reihe von Berufsfeldern mit Nachwuchsproblemen, macht das Gelingen solcher Maßnahmen zu einer dringlichen gesellschaftlichen Aufgabe. Im vorliegenden Aufsatz haben wir unsere Begleitstudien zu zwei Maßnahmen vorgestellt. In beiden Maßnahmen führten die fehlenden Deutschkompetenzen der Flüchtlinge zu oftmals gravierenden Verständigungsproblemen. Für die erste Phase der Immigration von Einwanderern ist das eine typische und erwartbare Situation, umso mehr a) als angesichts der großen Zahl von Fluchtmigranten innerhalb kurzer Zeitspanne nicht alle Zugewanderten schnell einen Sprachkursplatz bekommen können, b) die meisten Flüchtlinge keine Deutschkenntnisse mitbringen und c) ein erheblicher Prozentsatz kein hohes (sprachliches) Bildungsniveau aufweist. Aufgrund der häufig traumatischen Lebens- und Fluchtgeschichte fallen darüber hinaus Lernen und soziale Integration oft schwer, was sich ebenfalls negativ auf den Erwerb des Deutschen auswirkt.

Die hier vorgestellten Datenanalysen zeigen aber, dass eine Sicht, die sich einseitig auf die fehlenden und zu erwerbenden Sprach- und Interaktionskompetenzen der Flüchtlinge fokussiert, zu kurz greift. Berufliche Ausbildung ist ein interaktives Geschehen, dessen Verlauf und Erfolg von den Aktivitäten beider Seiten, der Ausbilder wie der Auszubildenden, abhängt. Wir konnten feststellen, dass den sprachlich-kommunikativen Praktiken des Instruierens und der Verständigungssicherung seitens der Ausbilder eine Schlüsselrolle für den Erfolg der Ausbildungsmaßnahmen zukommt. Als besonders wichtig erwiesen sich der Grad der Partnersensitivität des Instruierens und die Verbindung der fachlichen mit der sprachlichen Ausbildung. Partnersensitives Instruieren erfordert eine angemessene Einschätzung von Sprachkompetenzen und Fachwissen der Auszubildenden, die genaue Beobachtung und das responsive Eingehen auf das in der Interaktionssituation erkennbare Verständnis der Auszubildenden und die Fähigkeit zur adaptiven Formulierung und zur situationsflexiblen Nutzung von Mehrsprachigkeit. Die Einsicht, dass sprachliche Unterweisung ebenso wichtig ist wie die fachliche und mit Letzterer Hand in Hand gehen muss, ist nicht nur für das Verständlichmachen von Instruktionen, sondern auch für den Erwerb von Fähigkeiten der Kooperation im Team und generell den Erwerb situationstranszendenter Handlungsfähigkeiten entscheidend. Unsere Analysen zeigen, dass die Praktiken des Instruierens und der Verständigungssicherung in der L1-L2-Interaktion, die bereits von Forschern in anderen Ländern untersucht wurden, auch für die von uns betrachteten Ausbildungssettings grundlegend sind. Darüber hinaus konnten wir feststellen, dass illustrativen und Zeigegesten, der Verdeutlichung der jeweils anstehenden Handlungsaufgabe und der klaren Trennung zwischen verschiedenen Aktivitäten eine wichtige Rolle zukommt. Als besondere bereichsspezifische Vermittlungsaufgabe konnten wir die Beherrschung von Maßen und Zahlen feststellen. Die Verständigungssequenzen waren bezüglich referenzieller und thematischer Aspekte in der Regel erfolgreich, das genaue Verständnis der auszuführenden Aufgabe und komplexe prädikative Gehalte bereiteten dagegen oft Schwierigkeiten.

Die Ausbilder zeigten sich den Anforderungen an die Interaktionsgestaltung teils mehr („GASTRO“), teils weniger gewachsen („PerF Plus“). Es ließ sich feststellen, dass nicht nur mangelndes Problembewusstsein („PerF Plus“ Holzbau), sondern auch Hilflosigkeit 
und ungenügend passgenaue Partnersensitivität („PerF Plus“ Metallbau) Schwierigkeiten bereiteten. Gerade im letzteren Fall wurde sehr deutlich, dass Ausbilder wohl ein Bewusstsein dafür haben mögen, dass die Interaktion mit Flüchtlingen besondere sprachliche Anpassungsleistungen erfordert und in hohem Maße fragil ist, dass ihnen aber häufig die sprachlich-kommunikativen Kompetenzen fehlen, um in dieser Situation angemessen zu agieren. Untersuchungen wie die vorliegende sind daher notwendig, um zu einer empirisch fundierten Sprachsensibilisierung und sprachdidaktischen Ausbildung der Fachausbilder beizutragen, die unbedingt erforderlich ist, um den Erfolg der beruflichen Integrationsmaßnahmen sicherzustellen.

\section{Literatur}

Arbeitagentur (2017): Perspektiven für Flüchtlinge - PerF. Infoblatt. Online unter: www3 arbeitsagentur.de/ web/wcm/idc/groups/public/documents/webdatei/mdaw/mjk5/ edisp/16019022dstbai819996.pdf?_ba. sid=L6019022DSTBAI820002 (Stand 16.5.2018).

Auer, Peter (2013): Ethnische Marker im Deutschen zwischen Varietät und Stil. In: Deppermann, Arnulf (Hg.): Das Deutsch der Migranten. Berlin/New York: De Gruyter. S. 9-40. (= Jahrbuch des Instituts für Deutsche Sprache 2012).

Bechtel, Mark (2003): Interkulturelles Lernen beim Sprachenlernen in Tandem. Eine diskursanalytische Untersuchung. Tübingen: Narr.

Berg, Wilhelmine/Grünhage-Monetti, Matilde (2009): „Zur Integration gehört Spaß, Witz, Ironie, 'ne Sprache, die Firmensprache“. Sprachlich kommunikative Anforderungen am Arbeitsplatz. In: Deutsch als Zweitsprache 4. S. 7-21.

Brünner, Gisela (1987): Kommunikation in institutionellen Lehr-Lern-Prozessen. Diskursanalystische Untersuchung zu Instruktionen in der betrieblich Ausbildung. Tübingen: Narr. (= Kommunikation und Institution 16).

Bundesagentur für Arbeit (Hg.) (2017): Fluchtmigration. (= Berichte: Arbeitsmarkt kompakt). Online unter: https://statistik.arbeitsagentur.de/Statischer-Content/Statistische-Analysen/Statistische-Sonderberichte/ Generische-Publikationen/Fluchtmigration.pdf (Stand: 28.9.2017).

Cindark, Ibrahim (2010): Migration, Sprache und Rassismus. Der kommunikative Sozialstil der Mannheimer „Unmündigen“ als Fallstudie für die „emanzipatorischen Migranten“. Tübingen: Narr. (= Studien zur Deutschen Sprache 51).

Cindark, Ibrahim (im Dr.): Kommunikative Herausforderungen und Praktiken bei der Verständigung in beruflichen Maßnahmen für Flüchtlinge. In: Breindl, Eva et al. (Hg.): Proceedings of the 52nd Linguistics Colloquiums. Bern: Lang.

Cindark, Ibrahim/Hünlich, David (2017): Zur Gegenwart der sprachlich-beruflichen Integration von Flüchtlingen. Chancen und Risiken. In: Gross-Dinter, Ursula/Feuser, Florian/Ramos Méndez-Sahlende, Carmen (Hg.): Zum Umgang mit Migration. Zwischen Empörungsmodus und Lösungsorientierung. Bielefeld: transcript. S. 47-68. (= Edition Kulturwissenschaft 125).

Cindark, Ibrahim/Keim, Inken (2016): Von Gastarbeitern zu Transmigranten. Sprachliche Variation in deutsch-türkischen Lebenswelten. In: Ptashnyk et al. (Hg.). S. 219-243.

Cindark, Ibrahim/Ziegler, Evelyn (2016): Mehrsprachigkeit im Ruhrgebiet: Zur Sichtbarkeit sprachlicher Diversität in Dortmund. In: Ptashnyk et al. (Hg.). S. 133-156.

Clark, Herbert H. (1992): Arenas of Language Use. Chicago u.a.: University Press.

Clyne, Michael (1994): Inter-cultural communication at work. Cultural values in Discourses. Cambridge, UK: Cambridge University Press.

Dausendschön-Gay, Ulrich (1988): Particularités des réparations en situation de contact. In: Cosnier, Jacques/Gelas, Nadine/Kerbrat-Orecchioni, Catherine (Hg.): Echanges sur la conversation. Paris: CNRS. S. 269-284. 
Dausendschön-Gay, Ulrich/Gülich, Elisabeth/Krafft, Ullrich (1995): Exolinguale Kommunikation. In: Fiehler, Reinhard/Metzing, Dieter (Hg.): Untersuchungen zur Kommunikationsstruktur. Bielefeld: AisthesisVerlag. S. 85-117. (= Bielefelder Schriften zu Linguistik und Literaturwissenschaft 5).

Deppermann, Arnulf (2008): Verstehen im Gespräch. In: Kämper, Heidrun/Eichinger, Ludwig M. (Hg.): Sprache - Kognition - Kultur. Sprache zwischen mentaler Struktur und kultureller Prägung. Berlin/New York: De Gruyter. S. 225-261. (= Jahrbuch des Instituts für Deutsche Sprache 2007).

Deppermann, Arnulf (2016): La définition comme action multimodale pour des enjeux pratiques: définir pour instruire à l'auto-école. In: Langages 204, 4. S. 83-101.

Deppermann, Arnulf (2018): Instruction practices in German driving lessons. Differential uses of declaratives and imperatives. In: International Journal of Applied Linguistics. S. 1-18. Online unter: https://doi. org/10.1111/ijal.12198 (Stand 15.2.2018).

Deppermann, Arnulf/Schmidt, Axel (2016): Partnerorientierung zwischen Realität und Imagination. Anmerkungen zu einem zentralen Konzept der Dialogtheorie. In: Zeitschrift für Germanistische Linguistik 44, 3. S. 369-405.

Deppermann, Arnulf/Schmitt, Reinhold (2008): Verstehensdokumentation. Zur Phänomenologie von Verstehen in der Interaktion. In: Deutsche Sprache 8. S. 220-245.

Erickson, Frederick/Shultz, Jeffrey (1982): The counsellor as gatekeeper. Social interaction in interviews. New York: Academic Press.

Fasel Lauzon, Virginie (2014): Comprendre et apprendre dans l'interaction: les séquences d'explication en classe de français langue second. Bern: Lang.

Ferguson, Charles A. (1975): Toward a characterization of english foreigner talk. In: Anthropological Linguistics 17, 1. S. 1-14.

Firth, Alan (1996): The discursive accomplishment of normality. On 'lingua franca' English and conversation analysis. In: Journal of Pragmatics 26. S. 237-259.

Goffman, Erving (1955): On face-work. An analysis of ritual elements in social interaction. In: Psychiatry 18, 3. S. 213-231.

Grünhage-Monetti, Matilde (2009): Sprachlicher Bedarf von Personen mit Deutsch als Zweitsprache in Betrieben. Expertise im Auftrag des Bundesamtes für Migration und Flüchtlinge. Online unter: www. bamf.de/SharedDocs/Anlagen/DE/Publikationen/Expertisen/expertise-sprachlicher-bedarf.pdf?_blob= publicationFile (Stand 15.2.2018).

Grünhage-Monetti, Matilde (2013): Warum Deutsch nicht dort fördern, wo es gebraucht wird? Am Arbeitsplatz. In: Efing, Christian (Hg.): Ausbildungsvorbereitung im Deutschunterricht der Sekundarstufe I. Die sprachlich-kommunikativen Facetten von „Ausbildungsfähigkeit“. Frankfurt a.M.: Lang. S. 191-215.

Grünhage-Monetti, Matilde/Svet, Anna (2014): „... also ich glaube, das Reden ist das Allerwichtigste“. Kommunikation und berufliche Handlungskompetenz im Migrationskontext. In: Kiefer, Karl-Hubert et al. (Hg.): Berufsfeld-Kommunikation: Deutsch. Frankfurt a.M. u.a.: Lang. S. 177-198.

Gülich, Elisabeth/Kotschi, Thomas (1996): Textherstellungsverfahren in mündlicher Kommunikation. Ein Beitrag am Beispiel des Französischen. In: Motsch, Wolfgang (Hg.): Ebenen der Textstruktur: sprachliche und kommunikative Prinzipien. Tübingen: Niemeyer. S. 37-80. (= Reihe Germanistische Linguistik 164).

Gumperz, John J. (1982): Discourse strategies. Cambridge, UK: Cambridge University Press.

Gumperz, John J./Roberts, Celia (1991): Understanding in intercultural encounters. In: Blommaert, Jan/ Verschueren, Jef (Hg.): The pragmatics of international and intercultural communication. Amsterdam: Benjamins. S. 51-90.

Gumperz, John J./Jupp, Tom C./Roberts, Celia (1979): Crosstalk. A study of cross-cultural communication. London: National Centre for Industrial Language Training.

Günthner, Susanne (1993): Diskursstrategien in der interkulturellen Kommunikation. Analysen deutschchinesischer Gespräche. Tübingen: Niemeyer. (= Linguistische Arbeiten 286). 
Hazel, Spencer/Svennevig, Jan (2018): Multilingual workplaces. Interactional dynamics of the contemporary international workforce. In: Journal of Pragmatics 126. S. 1-9.

Hee, Katrin (2012): Polizeivernehmungen von Migranten. Eine gesprächsanalytische Studie interkultureller Interaktionen in Institutionen. Heidelberg: Winter. (= OraLingua 3).

Hindmarsh, Jon/Reynolds, Patricia/Dunne, Stephen (2011): Exhibiting understanding. The body in apprenticeship. In: Journal of Pragmatics 43, 2. S. 489-503.

Hinnenkamp, Volker (1982): Foreigner Talk und Tarzanisch: eine vergleichende Studie über die Sprechweise gegenüber Ausländern am Beispiel des Deutschen und des Türkischen. Hamburg: Buske.

Hinnenkamp, Volker (1989): Interaktionale Soziolinguistik und interkulturelle Kommunikation: Gesprächsmanagement zwischen Deutschen und Türken. Tübingen: Niemeyer. (= Linguistische Arbeiten 232).

Hinnenkamp, Volker (1998): Mißverständnisse in Gesprächen: eine empirische Untersuchung im Rahmen der interpretativen Soziolinguistik. Opladen/Wiesbaden: Westdt. Verlag.

House, Juliane (2010): The pragmatics of English as a lingua franca. In: Trosborg, Anna (Hg.): Handbook of Pragmatics. Bd. 7: Pragmatics Across Languages and Cultures. Berlin/Boston: De Gruyter. S. 363-387.

Iványi, Zsuzsanna (1998): Wortsuchprozesse. Eine gesprächsanalytische Untersuchung und ihre wissenschaftsmethodologischen Konsequenzen. Frankfurt a.M.: Lang.

Jefferson, Gail (1972): Side sequences. In: Sudnow, David (Hg.): Studies in social interaction. New York: Free Press. S. 294-338.

Kameyama, Shinichi (2004): Verständnissicherndes Handeln. Zur reparativen Bearbeitung von Rezeptionsdefiziten in deutschen und japanischen Diskursen. Münster: Waxmann.

Keim, Inken (2008): Die „türkischen Powergirls“. Lebenswelt und kommunikativer Stil einer Migrantinnengruppe in Mannheim. 2. durchges. Aufl. Tübingen: Narr. (= Studien zur deutschen Sprache 39).

Kendon, Adam (2004): Gesture. Cambridge, UK: Cambridge University Press.

Kern, Friederike (2000): Kultur(en) der Selbstdarstellung. Ost- und Westdeutsche in Bewerbungsgesprächen. Wiesbaden: Deutscher Universitätsverlag.

Koole, Tom (2012): Teacher evaluations. Assessing 'knowing', 'understanding' and 'doing'. In: Rasmussen, Gitte/Brouwer, Catherine E./Day, Denis (Hg.): Evaluating Cognitive Competences in Interaction. Amsterdam: Benjamins. S. 43-66.

Kurhila, Salla (2001): Correction in talk between native and non-native speaker. In: Journal of Pragmatics, 33. S. $1083-1110$

Kurhila, Salla (2006): Second Language Interaction. Amsterdam: Benjamins.

Lindwall, Oskar/Ekström, Anna (2012): Instruction in interaction. The teaching and learning of a manual skill. In: Human Studies 35, 1. S. 27-49.

Lindwall, Oskar/Lymer, Gustav/Greiffenhagen, Christian (2015): The sequential analysis of instruction. In: Numa Markee (Hg.): The handbook of classroom discourse and interaction. New York: Wiley. S. $142-157$.

Lüdi, Georges/Höchle, Katharina/Yanaprasart, Patchareerat (2010): Plurilingual practices at multilingual worklplaces. In: Meyer/Apfelbaum (Hg.). S. 211-234.

Marsh, David (1994): Bilingual education and content and language integrated learning. Paris: International Association for Cross-cultural Communication, Language Teaching in the Member States of the European Union (Lingua), University of Sorbonne.

Mazeland, Harrie/Zaman-Zadeh, Minna (2004): The logic of clarification. Some observations about wordclarification repairs in Finnish-as-a-lingua-franca interactions. In: Gardner, Roy/Wagner, Johannes (Hg.): Second Language Conversations. London/New York: Continuum. S. 132-156

Mehan, Hugh (1979): Learning lessons. Social organization in the classroom. Cambridge, UK: Cambridge University Press.

Meyer, Bernd (2004): Dolmetschen im medizinischen Aufklärungsgespräch. Eine diskursanalytische Untersuchung zur Arzt-Patienten-Kommunikation im mehrsprachigen Krankenhaus. Münster: Waxmann. 
Meyer, Bernd/Apfelbaum, Birgit (Hg.) (2010): Multilingualism at work. From policies to practices in public, medical, and business settings. Amsterdam u.a.: Benjamins.

Meyer, Bernd et al. (2010): Nurses as interpreters. Aspects of interpreter training for bilingual medical employees. In: Meyer/Apfelbaum (Hg.). S. 163-184.

Mol, Lisette et al. (2012): Adaptation in gesture. Converging hands or converging minds? In: Journal of Memory and Language 66. S. 249-264.

Mondada, Lorenza (2004): Ways of 'doing being plurilingual' in international work weetings. In: Gardner, Roy/Wagner, Johannes (Hg.): Second Language Conversations. London: Continuum. S. 27-60.

Mondada, Lorenza (2011): Understanding as an embodied, situated and sequential achievement in interaction. In: Journal of Pragmatics 43, 2. S. 542-552.

Mori, Junko/Hayashi, Makoto (2006): The achievement of intersubjectivity through embodied completions. A study of interactions between first and second language speakers. In: Applied Linguistics 27, 2. S. $195-219$.

Mortensen, Kristian (2011): Doing word explanation in interaction. In: Pallotti, Gabriela/Wagner, Johannes (Hg.): L2 learning as social practice: conversation-analytic perspectives. Honolulu: National Foreign Language Resource Center. S. 135-163.

Müller, Cornelia (1998): Redebegleitende Gesten. Kulturgeschichte - Theorie - Sprachvergleich. Berlin: Berlin Verlag.

Ptashnyk, Stefaniya et al. (Hg.) (2016): Gegenwärtige Sprachkontakte im Kontext der Migration. Heidelberg: Winter. (= Schriften des Europäischen Zentrums für Sprachwissenschaften 5).

Roberts, Celia (2007): Multilingualism in the workplace. In: Auer, Peter/Wei, Li (Hg.): Handbook of Multilingualism and Multilingual Communication. Berlin/New York: De Gruyter. S. 405-422. (= Handbooks of applied linguistics 5).

Roberts, Celia (2013): The gatekeeping of Babel. Job interviews and the linguistic penalty. In: Duchêne, Alexandre/Moyer, Melissa/Roberts, Celia (Hg.): Language, migration and social inequalities. A critical sociolinguistic perspective on institutions and work. Bristol: Multilingual Matters. S. 81-94.

Roberts, Celia/Davies, Eyelyn/Jupp, Thomas C. (1992): Language and discrimination: a study of communication in multi-ethnic workplaces. London u.a.: Longman.

Römhild, Regina/Vertovec, Steven (2009): Entwurf eines Integrations- und Diversitätskonzepts für die Stadt Frankfurt am Main. Ein Arbeitspapier des Dezernats für Integration. Online unter: www.frankfurt.de/ sixcms/media.php/738/Integrationskonzept.pdf (Stand 15.2.2018).

Rosenberg, Katharina (2014): Interkulturelle Behördenkommunikation. Eine gesprächsanalytische Untersuchung zu Verständigungsproblemen zwischen Migranten und Behördenmitarbeitern in Berlin und Buenos Aires. Berlin/Boston: De Gruyter.

Sacks, Harvey (1992): Lectures on conversation. Bd. 1. Oxford: Blackwell.

Sarangi, Srikant/Roberts, Celia (1999): Hybridity in gatekeeping discourse. Issues of practical relevance for the researcher. In: Sarangi, Srikant/Roberts, Celia (Hg.): Talk, work and institutional order. Berlin: De Gruyter. S. 473-503.

Schegloff, Emanuel A./Jefferson, Gail/Sacks, Harvey (1977): The preference for self-correction in the organization of repair in conversation. In: Language 53, 2. S. 361-82.

Schön, Almut (2012): Arzt-Patienten-Gespräche als L2-L1-Kommunikation. Eine Diskursanalyse zu Deutsch als Fremd- und Zweisprache im Beruf. Frankfurt a.M. u.a.: Lang. (= Werkstattreihe Deutsch als Fremdsprache 86).

Streeck, Jürgen (2009): Gesturecraft. The manu-facture of meaning. Amsterdam: Benjamins.

Svennevig, Jan (2004): Other-repetition as display of hearing, understanding and emotional stance. In: Discourse Studies 6, 4. S. 489-516.

Svennevig, Jan (2015): Én porsjon om gangen. Multimodal koordinering i samtaler med andrespråksbrukere. In: Engebretsen, Martin (Hg.): Det tredje språket. Multimodale studier av interkulturell kommunikasjon i kunst, skole og samfunnsliv. Kristiansand: Porta. S. 196-211. 
Svennevig, Jan (2018): "What's it called in Norwegian?" Acquiring L2 vocabulary items in the workplace. In: Journal of Pragmatics 126. Online unter: www.sciencedirect.com/science/article/pii/S03782166 17302370?via\%3Dihub (Stand 23.5.2018).

Svennevig, Jan et al. (2017): Pre-empting understanding problems in L1/L2 conversations. Evidence of effectiveness from simulated emergency calls. In: Applied Linguistics amx023. Online unter: https://doi. org/10.1093/applin/amx021 (Stand 15.2.2018).

Tranekjær, Louise (2015): Interactional categorization and gatekeeping. Institutional encounters with otherness. Bristol: Multilingual Matters.

Vertovec, Steven (2007): Super-diversity and its implications. In: Ethnic and Racial Studies 30, 6. S. 1024-1054.

Yasui, Eiko (2013): Collaborative idea construction. Repetition of gestures and talk in joint brainstorming. In: Journal of Pragmatics 46, 1. S. 157-172.

Wiese, Heike (2012): Kiezdeutsch. Ein neuer Dialekt entsteht. 2. durchges. Aufl. München: Beck.

Prof. Dr. Arnulf Deppermann/Dr. Ibrahim Cindark

Institut für Deutsche Sprache

R 5, 6-13

68161 Mannheim

E-Mail: deppermann@ids-mannheim.de/cindark@ids-mannheim.de 